\title{
On the Representation Theory of Virasoro Nets
}

\author{
SEBASTIANO CARPI* \\ Dipartimento di Scienze \\ Università "G. d'Annunzio" di Chieti-Pescara \\ Viale Pindaro 42, I-65127 Pescara, Italy \\ e-mail carpi@sci.unich.it
}

\begin{abstract}
We discuss various aspects of the representation theory of the local nets of von Neumann algebras on the circle associated with positive energy representations of the Virasoro algebra (Virasoro nets). In particular we classify the local extensions of the $c=1$ Virasoro net for which the restriction of the vacuum representation to the Virasoro subnet is a direct sum of irreducible subrepresentations with finite statistical dimension (local extensions of compact type). Moreover we prove that if the central charge $c$ is in a certain subset of $(1, \infty)$, including $[2, \infty)$, and $h \geq(c-1) / 24$, the irreducible representation with lowest weight $h$ of the corresponding Virasoro net has infinite statistical dimension. As a consequence we show that if the central charge $c$ is in the above set and satisfies $c \leq 25$ then the corresponding Virasoro net has no proper local extensions of compact type.
\end{abstract}

\section{Introduction}

The idea that the formulation of relativistic quantum physics in terms of local nets of von Neumann algebras (see e.g. [27]) provides a natural framework for the classification of two-dimensional conformal field theories was already

\footnotetext{
*Supported in part by the Italian MIUR and GNAMPA-INDAM.
} 
present in the late eighties in the work of Buchholz, Mack and Todorov [3]. As an illustration of this idea these authors classified the local conformal nets over $S^{1}$ (compactified light ray) whose common "germ" is the U(1) chiral current algebra, namely the local nets extending the one generated by a $\mathrm{U}(1)$ current. In the same paper they suggested the more general (and ambitious) classification program of conformal field theories $S^{1}$ whose "germ" is the Virasoro algebra Vir. In other words, they proposed to classify the local extensions of the Virasoro nets, i.e. the local nets of von Neumann algebras on $S^{1}$ which are generated by the positive energy unitary irreducible representations with lowest weight 0 (vacuum representations) of Vir or equivalently (see 45]) by a chiral energy-momentum tensor $T(z)$, cf. [5. Since the equivalence class of a Virasoro net is completely determined by the value of the central charge $c$ in the corresponding representation of Vir, one has to classify the local extensions of a family of nets labelled by a positive real number $c$ and this is a clearly well defined problem which in fact turns out to be equivalent to the one of classifying diffeomorphism covariant nets on the circle.

In a recent remarkable paper [33] Kawahigashi and Longo have been able to solve the above problem for all the Virasoro nets with $c<1$ and subsequently they used this result to classify all local conformal nets on the two-dimensional Minkowski space-time, having parity symmetry and central charge less then 1 [34]. The extension of their results in the $c \geq 1$ region appears to be a very important and difficult challenge.

In the transition from $c<1$ to $c \geq 1$ two drastic differences are immediately evident. The first is that the Virasoro nets with $c \geq 1$ are all known to be non-rational. The second is that they are all expected to have irreducible sectors with infinite statistical dimension [49, a fact that has been proved by the present author in the case $c=1$ [8]. Rationality and the absence of irreducible sectors with infinite statistical dimension play a fundamental role in the classification of $c<1$ conformal nets and hence, some of the main ideas in 33 do not apply for the remaining values of the central charge.

The purpose of this paper is give some new insight in the understanding of the representation theory of $c \geq 1$ Virasoro nets and their local extensions with the above problems in mind, especially concerning the role of infinite statistical dimension. Our main results are the classification of local extensions of compact type (see Definition [3.2) for the Virasoro net with $c=1$ 
(Theorem [3.5) ${ }^{1}$ and the proof that if the central charge $c$ is in a certain subset of $(1, \infty)$ containing $[2, \infty)$, then the irreducible positive energy representations with lowest weight $h \geq(c-1) / 24$ of the corresponding Virasoro net have infinite statistical dimension (Theorem 5.1 ), a fact that it is expected to hold for every $c>1$ and $h>0$ [49. As a consequence of the latter result we show that if $c \in[2,25]$ then the corresponding Virasoro net as no proper local extensions of compact type (Theorem [5.7). As in the $c=1$ case [8, we use oscillator (Fock) representations of Vir to obtain the result on infinite statistical dimension but the argument we have found for $c>1$ is more intricate and relies in part on recent results of S. Köster [38].

Besides these main results we also provide the proof of some relevant properties of the Virasoro nets which seem to appear at most implicitly in the literature, like the fact that every irreducible positive energy representation of a Virasoro net on a separable Hilbert space comes from a representation of the Virasoro algebra (Prop. 2.1) or the fact that every local extension of a Virasoro net is diffeomorphism covariant (Prop. 3.7).

\section{Preliminaries}

\subsection{Conformal nets on $S^{1}$ and diffeomorphism covari- ance}

Let $\mathcal{J}$ be the set of nonempty, nondense, open intervals of the unit circle $S^{1}=\{z \in \mathbb{C}:|z|=1\}$.

A conformal net on $S^{1}$ is a family $\mathcal{A}=\{\mathcal{A}(I): I \in \mathcal{J}\}$ of von Neumann algebras, acting on a infinite-dimensional separable complex Hilbert space $\mathcal{H}_{\mathcal{A}}$, satisfying the following properties:

(i) Isotony.

$$
\mathcal{A}\left(I_{1}\right) \subset \mathcal{A}\left(I_{2}\right), \text { if } I_{1} \subset I_{2}, I_{1}, I_{2} \in \mathcal{J} .
$$

(ii) Locality.

$$
\mathcal{A}\left(I_{1}\right) \subset \mathcal{A}\left(I_{2}\right)^{\prime}, \text { if } I_{1} \cap I_{2}=\emptyset, I_{1}, I_{2} \in \mathcal{J} .
$$

(iii) Möbius covariance. There exists a strongly continuous unitary representation $U$ of $\operatorname{PSL}(2, \mathbb{R})$ in $\mathcal{H}$ such that

$$
U(g) \mathcal{A}(I) U(g)^{-1}=\mathcal{A}(g I), I \in \mathcal{J}, g \in \operatorname{PSL}(2, \mathbb{R}),
$$

\footnotetext{
${ }^{1}$ An equivalent result as been independently obtained by $\mathrm{F} . \mathrm{Xu}$ [55]
} 
where $\operatorname{PSL}(2, \mathbb{R})$ acts on $S^{1}$ by Möbius transformations (cf. Appendix A).

(iv) Positivity of the energy. The representation $U$ has positive energy, namely the conformal Hamiltonian $L_{0}$, which generates the restriction of $U$ to the one-parameter subgroup of rotations $r(\vartheta)$, has nonnegative spectrum.

(v) Existence and uniqueness of the vacuum. There exists a unique (up to aphase) $U$-invariant unit vector $\Omega \in \mathcal{H}_{\mathcal{A}}$.

(vi) Cyclicity of the vacuum. $\Omega$ is cyclic for the algebra $\mathcal{A}\left(S^{1}\right):=\bigvee_{I \in \mathcal{J}} \mathcal{A}(I)$

Some consequences of the axioms are [22, 25, 19]:

(vii) Reeh-Schlieder property. For every $I \in \mathcal{J}, \Omega$ is cyclic and separating for $\mathcal{A}(I)$.

(viii) Bisognano-Wichmann property. If $\Delta_{I}$ is the modular operator associated to $\mathcal{A}(I)$ and $\Omega$ then

$$
\Delta_{I}^{i t}=U\left(\Lambda_{I}((2 \pi) t)\right.
$$

where $\Lambda_{I}$ is the one parameter subgroup of $\operatorname{PSL}(2, \mathbb{R})$ of special conformal transformations preserving $I$.

(ix) Haag duality. For every $I \in \mathcal{J}$

$$
\mathcal{A}(I)^{\prime}=\mathcal{A}\left(I^{c}\right),
$$

where $I^{c}$ denotes the interior of $S^{1} \backslash I$.

(x) Factoriality. The algebras $\mathcal{A}(I)$ are type $\mathrm{III}_{1}$ factors.

(xi) Irreducibility. $\mathcal{A}\left(S^{1}\right)=\mathrm{B}\left(\mathcal{H}_{\mathcal{A}}\right)$, where $\mathrm{B}\left(\mathcal{H}_{\mathcal{A}}\right)$ denotes the algebra of all bounded linear operators on $\mathcal{H}_{\mathcal{A}}$.

(xii) Additivity. If $\mathcal{S} \subset \mathcal{J}$ is a covering of the interval $I$ then

$$
\mathcal{A}(I) \subset \bigvee_{J \in \mathcal{S}} \mathcal{A}(J)
$$


Furthermore, it follows easily from the strong continuity of the representation $U$ that conformal nets are continuous from outside, namely

$$
\mathcal{A}(I)=\bigcap_{J \supset \bar{I}} \mathcal{A}(J) .
$$

A conformal net $\mathcal{A}$ is said to be split if given two intervals $I_{1}, I_{2} \in \mathcal{J}$ such that the closure $\overline{I_{1}}$ of $I_{1}$ is contained in $I_{2}$, there exists a type I factor $\mathcal{N}\left(I_{1}, I_{2}\right)$ such that

$$
\mathcal{A}\left(I_{1}\right) \subset \mathcal{N}\left(I_{1}, I_{2}\right) \subset \mathcal{A}\left(I_{2}\right) .
$$

If $\operatorname{Tr}\left(t^{L_{0}}\right)<\infty$ for every $t \in(0,1)$ then $\mathcal{A}$ is split [13, Theorem 3.2].

$\mathcal{A}$ is said to be strongly additive if for every $I, I_{1}, I_{2} \in \mathcal{J}$ with $I_{1}, I_{2}$ obtained by removing a point from $I$ we have

$$
\mathcal{A}\left(I_{1}\right) \vee \mathcal{A}\left(I_{2}\right)=\mathcal{A}(I) .
$$

It is often convenient to identify $S^{1} /\{-1\}$ with the real line $\mathbb{R}$. With this identification, the family of nonempty open bounded intervals of $\mathbb{R}$ corresponds to the family $\mathcal{J}_{0}=\{I \in \mathcal{J}:-1 \notin \bar{I}\}$. The restriction $\mathcal{A}_{0}$ of a conformal net $\mathcal{A}$ to $\mathcal{J}_{0}$ can be considered as a net on $\mathbb{R}$. Moreover, since $\mathcal{J}_{0}$ is directed under inclusion, one can define the quasi-local $C^{*}$-algebra (still denoted $\mathcal{A}_{0}$ ) $\left.\left(\bigcup_{I \in J_{0}} \mathcal{A}(I)\right)^{-\|\cdot\|}\right)$ as $C^{*}$-inductive limit of the local von Neumann algebras $\mathcal{A}(I), I \in \mathcal{J}_{0}$.

We now briefly discuss diffeomorphism covariance. Let $\operatorname{Diff}^{+}\left(S^{1}\right)$ the group of orientation preserving diffeomorphisms of the circle. It is an infinite dimensional Lie group modelled on the real topological vector space $\operatorname{Vect}\left(S^{1}\right)$ of smooth real vectors fields on $S^{1}$ with the usual $C^{\infty}$ topology [46, Sect.6]. Its Lie algebra coincides with $\operatorname{Vect}\left(S^{1}\right)$ with the bracket given by the negative of the usual brackets of vector fields. Hence if $g(z), f(z), z=e^{i \vartheta}$, are real valued functions in $C^{\infty}\left(S^{1}\right)$ then

$$
\left[g\left(e^{i \vartheta}\right) \frac{d}{d \vartheta}, f\left(e^{i \vartheta}\right) \frac{d}{d \vartheta}\right]=\left(\frac{d}{d \vartheta} g\left(e^{i \vartheta}\right)\right) f\left(e^{i \vartheta}\right)-\left(\frac{d}{d \vartheta} f\left(e^{i \vartheta}\right)\right) g\left(e^{i \vartheta}\right) .
$$

In this paper we shall often identify the vector field $g\left(e^{i \vartheta}\right) \frac{d}{d \vartheta} \in \operatorname{Vect}\left(S^{1}\right)$ with the corresponding real function $g(z) \in C^{\infty}\left(S^{1}\right)$.

Following [33] for every $I \in \mathcal{J}$ we shall denote by $\operatorname{Diff}(I)$ the subgroup of $\operatorname{Diff}^{+}\left(S^{1}\right)$ whose elements are the diffeomorphisms of the circle which act as the identity on $I$. Note that $\operatorname{Diff}(I)$ does not coincide with the group of 
diffeomorphisms of the open interval $I$, as the notation might erroneously suggest.

By a strongly continuous projective unitary representation $V$ of $\operatorname{Diff}^{+}\left(S^{1}\right)$ on a Hilbert space we shall always mean a strongly continuous homomorphism of $\operatorname{Diff}^{+}\left(S^{1}\right)$ into the quotient $\mathrm{U}(\mathcal{H}) / \mathbb{T}$ of the unitary group of $\mathcal{H}$ by the circle subgroup $\mathbb{T}$. The restriction of the representation $V$ to the Möbius subgroup of $\operatorname{Diff}^{+}\left(S^{1}\right)$ always lifts to a unique strongly continuous unitary representation $U$ of the universal covering group $\widehat{\operatorname{PSL}(2, \mathbb{R})}$ of $\operatorname{PSL}(2, \mathbb{R})$. We shall say that $V$ extends $U$ and that $V$ is a positive energy representation if $U$ is a positive energy representation of $\widehat{\operatorname{PSL}(2, \mathbb{R})}$, namely if the corresponding conformal Hamiltonian $L_{0}$, which generates the restriction of $U$ to the lifting $\tilde{r}(\vartheta)$ of the one-parameter subgroup $r(\vartheta)$ of rotations, has nonnegative spectrum. Note that although for $\gamma \in \operatorname{Diff}^{+}\left(S^{1}\right), V(\gamma)$ is defined only up to a phase as an operator on $\mathcal{H}$, expressions like $V(\gamma) T V(\gamma)^{*}$ for $T \in \mathrm{B}(\mathcal{H})$ or $V(\gamma) \in \mathcal{M}$ for a (complex) subspace $\mathcal{M} \subset \mathrm{B}(\mathcal{H})$ are unambiguous and will be used in the following.

We shall say that a conformal net on $S^{1}$ is diffeomorphism covariant if there is a strongly continuous projective unitary representation $V$ of $\operatorname{Diff}^{+}\left(S^{1}\right)$ on $\mathcal{H}_{\mathcal{A}}$ extending $U \circ q$ ( where $U$ is the original unitary representation of $\operatorname{PSL}(2, \mathbb{R})$ making $\mathcal{A}$ Möbius covariant and $q: \widetilde{\operatorname{PSL}(2, \mathbb{R})} \mapsto \operatorname{PSL}(2, \mathbb{R})$ denotes the covering map) and such that, for every $I \in \mathcal{J}$

$$
\begin{gathered}
V(\gamma) \mathcal{A}(I) V(\gamma)^{*}=\mathcal{A}(\gamma I), \gamma \in \operatorname{Diff}^{+}\left(S^{1}\right), \\
V(\gamma) A V(\gamma)^{*}=A, \gamma \in \operatorname{Diff}(I), A \in \mathcal{A}\left(I^{c}\right) .
\end{gathered}
$$

\subsection{Representations of conformal nets}

A representation of a conformal net $\mathcal{A}$ is a family $\pi=\left\{\pi_{I}: I \in \mathcal{J}\right\}$ where $\pi_{I}$ is a (unital) representation of $\mathcal{A}(I)$ on a fixed Hilbert space $\mathcal{H}_{\pi}$, such that

$$
\left.\pi_{J}\right|_{\mathcal{A}(I)}=\pi_{I} \text { if } I \subset J, I, J \in \mathcal{J} .
$$

Irreducibility, direct sums and unitary equivalence of representations of a conformal net can be defined in a natural way, see [22, 25].

If $\mathcal{H}_{\pi}$ is separable then, since the local von Neumann algebras $\mathcal{A}(I), I \in \mathcal{J}$, are factors, $\pi$ is automatically locally normal, namely $\pi_{I}$ is normal for each $I \in \mathcal{J}$, see 51 . Hence, $\pi_{I}(\mathcal{A}(I))$ is a type $\mathrm{III}_{1}$ factor. The unitary equivalence class a representation $\pi$ on a separable Hilbert space is called a sector 
and denoted $[\pi]$. If $\pi$ is irreducible then we say that $[\pi]$ is an irreducible sector (also called superselection sector). The defining representation $\pi_{0}$ of a conformal net $\mathcal{A}$ on the Hilbert space $\mathcal{H}_{\mathcal{A}}$ is called the vacuum representation. The corresponding sector is called the vacuum sector and $\mathcal{H}_{\mathcal{A}}$ is said to be the vacuum Hilbert space of $\mathcal{A}$.

A representation $\pi$ is said to be covariant if there is a strongly continuous unitary representation $U_{\pi}$ of $\operatorname{PSL}(2, \mathbb{R})$ on $\mathcal{H}_{\pi}$ such that

$$
\operatorname{Ad} U_{\pi}(g) \circ \pi_{I}=\pi_{q(g) I} \circ \operatorname{Ad} U(q(g)), g \in \widetilde{\operatorname{PSL}(2, \mathbb{R})}, I \in \mathcal{J} .
$$

If $U_{\pi}$ can be chosen to be a positive energy representation, then $\pi$ is said to be covariant with positive energy. In this case one can always choose $U_{\pi}$ to be with positive energy and inner, namely such that

$$
U_{\pi}(\widetilde{\operatorname{PSL}(2, \mathbb{R})}) \subset \pi(\mathcal{A})^{\prime \prime}:=\bigvee_{I \in \mathcal{J}} \pi_{I}(\mathcal{A}(I)),
$$

and this choice is unique, see [36] and (the proof of) [1, Lemma 5.14].

Given a covariant representation $\pi$ of $\mathcal{A}$ on a separable Hilbert space $\mathcal{H}_{\pi}$ one has the (isomorphic) type III subfactors $\pi_{I}(\mathcal{A}(I)) \subset \pi_{I^{c}}\left(\mathcal{A}\left(I^{c}\right)\right)^{\prime}, I \in$ $\mathcal{J}$ [22. Hence the corresponding (minimal) index $\left[\pi_{I^{c}}\left(\mathcal{A}\left(I^{c}\right)\right)^{\prime}: \pi_{I}(\mathcal{A}(I))\right]$ [29, 39, 41] is independent of $I \in \mathcal{J}$ and the statistical dimension $d(\pi)$ of $\pi$ is given by

$$
d(\pi)=\left[\pi_{I^{c}}\left(\mathcal{A}\left(I^{c}\right)\right)^{\prime}: \pi_{I}(\mathcal{A}(I))\right]^{\frac{1}{2}} .
$$

A representation $\rho$ of a conformal net $\mathcal{A}$ on its vacuum Hilbert space $\mathcal{H}_{\mathcal{A}}$ is said to be localized in an interval $I_{0} \in \mathcal{J}$ if $\rho_{I_{0}^{c}}$ is the identity representation. As a consequence of Haag duality if a representation $\rho$ is localized in $I_{0}$ and $I \in \mathcal{J}$ contains $I_{0}$ then $\rho_{I}$ is an endomorphism of $\mathcal{A}(I)$ whose index is the square of the statistical dimension of the representation $\rho$. Moreover, for every interval $I_{0} \in \mathcal{J}$ and every representation $\pi$ of $\mathcal{A}$ on a separable Hilbert space one can find a representation $\rho$, localized in $I_{0}$ and unitarily equivalent to $\pi$, see [22, 25]. The restriction to $\mathcal{A}_{0}$ of a representation $\rho$ localized in some $I_{0} \in J_{0}$ is called a DHR endomorphism and in fact yields an endomorphism of the quasi-local $C^{*}$-algebra $\mathcal{A}_{0}$, see [43, Sect. 3]. The set of DHR endomorphisms is a semigroup (under composition) and it has a natural (DHR) unitary braiding, see [20, 22. As usual we shall denote $\epsilon(\rho, \sigma)$ the unitary braiding operator associated to the DHR endomorphisms $\rho$ and $\sigma$. 


\subsection{Subsystems}

A conformal subsystem (or subnet) of a conformal net $\mathcal{A}$ is a family $\mathcal{B}=\{\mathcal{B}(I): I \in \mathcal{J}\}$ of nontrivial von Neumann algebras acting on $\mathcal{H}_{\mathcal{A}}$ such that:

$$
\begin{array}{r}
\mathcal{B}(I) \subset \mathcal{A}(I) I \in \mathcal{J} ; \\
U(g) \mathcal{B}(I) U(g)^{-1}=\mathcal{B}(g I) I \in \mathcal{J}, g \in \operatorname{PSL}(2, \mathbb{R}) ; \\
\mathcal{B}\left(I_{1}\right) \subset \mathcal{B}\left(I_{2}\right) \text { if } I_{1} \subset I_{1}, \quad I_{1}, I_{2} \in \mathcal{J} .
\end{array}
$$

We shall use the notation $\mathcal{B} \subset \mathcal{A}$ for conformal subsystems. Note that $\mathcal{B}$ is not in general a conformal net since $\Omega$ is not cyclic for the algebra $\mathcal{B}\left(S^{1}\right):=\bigvee_{I \in \mathcal{J}} \mathcal{B}(I)$, unless $\mathcal{B}=\mathcal{A}$. However one gets a conformal net $\widehat{\mathcal{B}}$ restricting the algebras $\mathcal{B}(I), I \in \mathcal{J}$, and of the representation $U$ to the closure $\mathcal{H}_{\mathcal{B}}$ of $\mathcal{B}\left(S^{1}\right) \Omega$. Since the map $\left.b \in \mathcal{B}(I) \mapsto b\right|_{\mathcal{H}_{\mathcal{B}}} \in \widehat{\mathcal{B}}(I)$ is an isomorphism for every $I \in \mathcal{J}$, because of the Reeh-Schlieder property, as

usual, we shall often use the symbol $\mathcal{B}$ instead of $\widehat{\mathcal{B}}$, specifying, if necessary, when $\mathcal{B}$ acts on $\mathcal{H}_{\mathcal{A}}$ or on $\mathcal{H}_{\mathcal{B}}$.

Let $\pi$ be the defining representation of the conformal net $\mathcal{B} \subset \mathcal{A}$ on the Hilbert space $\mathcal{H}_{\mathcal{A}}$ (i.e. the restriction to $\mathcal{B}$ of the vacuum representation of $\mathcal{A}$ ). Because of the separability of $\mathcal{H}_{\mathcal{A}}$, for every $I_{0} \in \mathcal{J}$ we can find a representation $\theta$ on the vacuum Hilbert space $\mathcal{H}_{\mathcal{B}}$ of $\mathcal{B}$, which is unitarily equivalent to $\pi$ and is localized in $I_{0}$. Then if $I_{0} \subset I \in \mathcal{J}, \theta_{I}$ is a dual canonical endomorphism for the subfactor $\mathcal{B}(I) \subset \mathcal{A}(I)$, namely there is a canonical endomorphism (in the sense of [41]) for the latter whose restriction to $\mathcal{B}(I)$ coincides with $\theta_{I}$, see [44, Proposition 3.4] and [43, Sect. 3.3].

\subsection{Virasoro nets and their representations}

Let Vir denote the Virasoro algebra i.e. the complex Lie algebra spanned by $L_{n}, n \in \mathbb{Z}$ and a central element $\kappa$ with relations

$$
\left[L_{n}, L_{m}\right]=(n-m) L_{n+m}+\delta_{n+m, 0} \frac{n^{3}-n}{12} \kappa .
$$

We shall denote $L(c, h)$ the unique positive energy irreducible unitary representation of Vir with lowest weight $h$ and central charge $c$ (see e.g. [14, 30]). The conformal Hamiltonian $L_{0}$ is diagonalizable on the corresponding Virmodule (still denoted $L(c, h)$ ) with spectrum $h+\mathbb{N}_{0}$ and the central element 
$\kappa$ acts as multiplication by the real number $c$. Positivity of the energy implies $h \geq 0$ and unitarity (or hermiticity) means that there is a positive definite sesquilinear form $(\cdot, \cdot)$ on $L(c, h)$ such that

$$
\left(\xi, L_{n} \psi\right)=\left(L_{-n} \xi, \psi\right)
$$

for $\xi, \psi \in L(c, h), n \in \mathbb{Z}$.

The above conditions give restrictions on the values of the pair $(c, h)$. In fact either $c \geq 1$ and $h \geq 0$ or we have a pair $\left(c(m), h_{p, q}(m)\right), m \in \mathbb{N}$, where

$$
c(m)=1-\frac{6}{(m+2)(m+3)}
$$

and

$$
h_{p, q}(m)=\frac{((m+3) p-(m+2) q)^{2}-1}{4(m+2)(m+3)},
$$

$p=1, \ldots, m+1, q=1, \ldots, p$, (discrete series representations). For later convenience we shall denote $D \subset\left[\frac{1}{2}, 1\right)$ the set of discrete values of the central charge in Eq. (22). Accordingly the set of allowed values of the central charge is $D \cup[1, \infty)$.

Now let $\mathcal{H}(c, h)$ be the Hilbert space completion of the module $L(c, h)$. Then the Virasoro algebra acts on $\mathcal{H}(c, h)$ by unbounded operators on the common invariant domain $L(c, h) \subset \mathcal{H}(c, h)$ which can in fact be identified with the subspace $\mathcal{H}^{\text {in }}(c, h)$ of finite energy vectors i.e. the linear span of the eigenvectors of the conformal Hamiltonian. The (chiral) energy-momentum tensor $T_{(c, h)}(z), z=e^{i \vartheta} \in S^{1}$ associated to $L(c, h)$, is defined by the formal power series

$$
T_{(c, h)}(z)=\sum_{n \in \mathbb{Z}} L_{n} z^{-n-2}
$$

For a function on $S^{1}, \vartheta \mapsto f\left(e^{i \vartheta}\right)$ with finite Fourier series (trigonometric polynomial), the operator

$$
T_{(c, h)}(f)=\sum_{n \in \mathbb{Z}} L_{n} f_{n},
$$

where

$$
f_{n}=\int_{0}^{2 \pi} \frac{d \vartheta}{2 \pi} e^{-i n \vartheta} f\left(e^{i \vartheta}\right),
$$


belongs to Vir and hence is well defined on $\mathcal{H}^{\text {fin }}(c, h)$ and leave it invariant. Also the following (formal) notation is used

$$
T_{(c, h)}(f)=\oint_{S^{1}} \frac{z d z}{2 \pi i} T_{(c, h)}(z) f(z)=\int_{0}^{2 \pi} \frac{d \vartheta}{2 \pi} T_{(c, h)}\left(e^{i \vartheta}\right) e^{i 2 \vartheta} f\left(e^{i \vartheta}\right) .
$$

The Virasoro net $\mathcal{A}_{(\mathrm{Vir}, c)}$ can be defined as in [5] as the net generated by the energy-momentum tensor $T_{c}(z):=T_{(c, 0)}(z)$ in the representation of lowest weight 0 on $\mathcal{H}(c, 0)=: \mathcal{H}_{\mathcal{A}_{(\mathrm{Vir}, c)}}$. First of all one can show that the map $f \mapsto T_{c}(f)$ extends (uniquely) to an operator valued distribution (Wightman field) on the invariant domain $C^{\infty}\left(L_{0}\right)$, the subspace of smooth vectors for $L_{0}$. Moreover the linear energy-bounds established in [5] (also cf. 24]) imply that for every smooth real valued function $f, T_{c}(f)$ is essentially self-adjoint (on any core for $L_{0}$ ) and that $e^{i T_{c}\left(f_{1}\right)}$ commutes with $e^{i T_{c}\left(f_{2}\right)}$ when the real smooth functions $f_{1}$ and $f_{2}$ have disjoint supports (in fact these properties also hold in the representations with $h>0$ ). It follows that the net of von Neumann algebras defined by

$$
\mathcal{A}_{(\operatorname{Vir}, c)}(I)=\left\{\mathrm{e}^{i T_{c}(f)}: f \in C^{\infty}\left(S^{1}\right) \text {, real, supp } f \subset I\right\}^{\prime \prime}, \quad I \in \mathcal{J} .
$$

is local and in fact one can verify all the other axioms of a conformal net. In particular the representation $U$ of $\operatorname{PSL}(2, \mathbb{R})$ is obtained by integrating the self-adjoint part of the (complex) Lie subalgebra of Vir spanned by $L_{-1}, L_{0}, L_{1}$ and the vacuum vector $\Omega$ is the (normalized) lowest weight vector in $L(c, 0)$.

An alternative construction is obtained by integrating the representations $L(c, 0)$ of Vir to the corresponding projective unitary representations of $\operatorname{Diff}^{+}\left(S^{1}\right)$. In fact as shown by Goodman and Wallach [24] (cf. also [53]), for each allowed pair $(c, h)$ there is a unique strongly continuous projective unitary representation $V_{(c, h)}$ of $\operatorname{Diff}^{+}\left(S^{1}\right)$ on $\mathcal{H}(c, h)$ satisfying

$$
V_{(c, h)}(\exp (f))=p\left(e^{i T_{(c, h)}(f)}\right)
$$

for every real smooth function (vector field) $f$ on $S^{1}$. Here $\exp (f) \in \operatorname{Diff}^{+}\left(S^{1}\right)$ denotes the exponential of the vector field $f$, namely $t \mapsto \exp (t f)$ is the unique one-parameter group of diffeomorphisms generated by $f$, and $p$ : $\mathrm{U}(\mathcal{H}(c, h)) \mapsto \mathrm{U}(\mathcal{H}(c, h)) / \mathbb{T}$ denotes the quotient map. Then the net $\mathcal{A}_{(\mathrm{Vir}, c)}$ can be defined by

$$
\mathcal{A}_{(\operatorname{Vir}, c)}(I)=\left\{V_{(c, 0)}(\gamma): \gamma \in \operatorname{Diff}(I)\right\}^{\prime \prime},
$$




\section{$I \in \mathcal{J}$.}

The two definitions are equivalent because the group generated by the exponentials of smooth vector fields with support in $I \in \mathcal{J}$ is dense in $\operatorname{Diff}(I)$, see [40, Sect. V.2]. From the second one the diffeomorphism covariance of the Virasoro nets is explicit.

As a consequence of the finiteness of the (vacuum) Virasoro characters $\chi(t):=\operatorname{Tr}\left(t^{L_{0}}\right)$ for every $t \in(0,1)$ the Virasoro nets are split for every allowed value of the central charge. For $c \leq 1$ the Virasoro nets are strongly additive 33. 55] while for $c>1$ they are not [5].

We now discuss some properties of the representation theory of the Virasoro nets that we shall need in the following. Let $\mathcal{H}(c, h)$ be the Hilbert space completion of Vir module $L(c, h)$ as at the beginning of this subsection and let $T_{(c, h)}(z)$ be the corresponding energy-momentum tensor. A representation of $\mathcal{A}_{(\mathrm{Vir}, c)}$ on $\mathcal{H}(c, h)$ will be denoted $\pi_{h}^{c}$ if for every $I \in \mathcal{J}$ and every real smooth real function $f$ on $S^{1}$ with support in $I$, the following hold

$$
\pi_{h I}^{c}\left(e^{i T_{c}(f)}\right)=e^{i T_{(c, h)}(f)} .
$$

It is immediate to verify that if a representation satisfying Eq. (31) exists, then it is unique. More complicate is to demonstrate the existence of such representations. Of course the vacuum representation $\pi_{0}^{c}$ exists for every allowed value of $c$ i.e. for each $c \in D \cup[1, \infty)$. If $c<1$ and $h$ is a corresponding allowed value of the lowest weight then the representation $\pi_{h}^{c}$ exists as a consequence of the Goddard, Kent, Olive coset construction [23] and the local equivalence of positive energy representations of the loop groups $L \mathrm{SU}(2)$ at fixed level [22, 54, cf. [40, V.3.3.2] and [33, Sect. 3]. If $c \geq 1$ the existence of $\pi_{h}^{c}$ has been proved by D. Buchholz and H. SchulzMirbach for every $h \geq(c-1) / 24$. Finally if $c \in(D+1) \cup[2, \infty)$, then $c-1$ is an allowed value of the central charge. Then using the embedding

$$
\mathcal{A}_{(\mathrm{Vir}, c)} \subset \mathcal{A}_{(\mathrm{Vir}, c-1)} \otimes \mathcal{A}_{(\mathrm{Vir}, 1)}
$$

one can easily construct, for every $h \geq 0$, the representation $\pi_{h}^{c}$ as a subrepresentation of the restriction to $\mathcal{A}_{(\mathrm{Vir}, c)}$ of $\pi_{0}^{c-1} \otimes \pi_{h}^{1}$. ${ }^{2}$ As far as we know the existence of the representation $\pi_{h}^{c}$ for the remaining allowed pairs $(c, h)$ is still an important open problem.

\footnotetext{
${ }^{2}$ I learned this argument in an unpublished manuscript of D. Buchholz $[6]$.
} 
Proposition 2.1. If $\pi$ is an irreducible covariant representation with positive energy of the Virasoro net $\mathcal{A}_{(\mathrm{Vir}, \mathrm{c})}$ on a separable Hilbert space $\mathcal{H}_{\pi}$ then it is unitarily equivalent to $\pi_{h}^{c}$ for some $h \geq 0$.

Proof. Let $V_{(c, 0)}$ be the unique projective unitary representation of $\operatorname{Diff}^{+}\left(S^{1}\right)$ on $\mathcal{H}_{\mathcal{A}_{(\mathrm{Vir}, \mathrm{c})}}$ such that Eq. (29) holds with $h=0$. From [37, Sect. 2] (cf. also [33, Lemma 3.1]) we know that there is a strongly continuous positive energy projective unitary representation $V_{\pi}$ of $\operatorname{Diff}^{+}\left(S^{1}\right)$ on $\mathcal{H}_{\pi}$ such that $p\left(\pi_{I}\left(V_{(c, 0)}(\gamma)\right)\right)=V_{\pi}(\gamma)$ for each $I \in \mathcal{J}$ and $\gamma \in \operatorname{Diff}(I)$. Then it follows from the irreducibility and local normality of $\pi$ that $V_{\pi}$ is irreducible. As a consequence of Theorem A.2 in the Appendix , there is on $\mathcal{H}_{\pi}^{\text {fin }}$ a positive energy representation $R_{\pi}$ of the Virasoro algebra with central charge $c^{\prime} \in$ $D \cup[1, \infty)$, which is unitarily equivalent to $L\left(c^{\prime}, h\right)$ for some $h \geq 0$. Let

$$
T^{\pi}(z)=\sum_{n \in \mathbb{Z}} L_{n}^{\pi} z^{-n-2}
$$

be the corresponding energy-momentum tensor. Then, for every real smooth vector field $f$ on $S^{1}$, we have

$$
V_{\pi}(\exp (f))=p\left(e^{i T^{\pi}(f)}\right) .
$$

It follows that if $I \in \mathcal{J}$ and the support of $f$ is contained in $I$

$$
\pi_{I}\left(e^{i T_{c}(f)}\right)=e^{i \alpha_{I}(f)} e^{i T^{\pi}(f)},
$$

where $\alpha_{I}(f)$ is a real constant. Now, it is fairly easy to check that there is a (necessarily unique ) distribution $\alpha$ such that $\alpha(f)=\alpha_{I}(f)$ for every $I \in \mathcal{J}$ and every real function $f$ with support in contained in $I$ and that Möbius covariance implies that $\alpha=0$. Hence we have the equality

$$
\pi_{I}\left(e^{i T_{c}(f)}\right)=e^{i T^{\pi}(f)},
$$

which implies $c^{\prime}=c$. The conclusion then follows because the representation $R_{\pi}$ of Vir is unitarily equivalent to $L(c, h)$ for some $h \geq 0$.

We conclude this subsection with the following proposition.

Proposition 2.2. Let $\pi$ be a positive energy covariant representation of the Virasoro net $\mathcal{A}_{(\mathrm{Vir}, \mathrm{c})}$ on a separable Hilbert space and let $U_{\pi}$ be the corresponding unique inner unitary representation of $\widetilde{\mathrm{PSL}(2, \mathbb{R})}$. Assume that $U_{\pi}(\tilde{r}(2 \pi)) \in \mathbb{C} 1$. Then the following hold: 
(a) The representation $\pi$ is a direct sum of irreducible covariant positive energy representations.

(b) There exists a unique strongly continuous projective unitary representation $V_{\pi}$ of $\operatorname{Diff}^{+}\left(S^{1}\right)$ on $\mathcal{H}_{\pi}$ satisfying

$$
p\left(\pi_{I}\left(e^{i T_{c}(f)}\right)\right)=V_{\pi}(\exp (f)),
$$

for every $I \in \mathcal{J}$ and every real smooth function $f$ with support contained in I. Moreover, this representation satisfies

$$
\begin{array}{r}
V_{\pi}(\gamma) \in \pi_{I}\left(\mathcal{A}_{(\mathrm{Vir}, c)}(I)\right) \quad \forall I \in \mathcal{J}, \forall \gamma \in \widetilde{\operatorname{Diff}(I)}, \\
V_{\pi}(q(g))=p\left(U_{\pi}(g)\right) \quad \forall g \in \widehat{\operatorname{PSL}(2, \mathbb{R})} .
\end{array}
$$

Proof. The net $\mathcal{A}_{(\mathrm{Vir}, \mathrm{c})}$ has the split property and hence, has a consequence of [35. Proposition 56], $\pi$ has a direct integral decomposition

$$
\pi=\int_{X}^{\oplus} \pi_{\lambda} d \mu(\lambda),
$$

where, for almost every $\lambda, \pi_{\lambda}$ is an irreducible representation of $\mathcal{A}_{(\mathrm{Vir}, c)}$ on a separable Hilbert space $\mathcal{H}(\lambda)$. Since $U_{\pi}(g) \in \pi\left(\mathcal{A}_{(\mathrm{Vir}, c)}\right)$ " for each $g \in$ $\operatorname{PSL}(2, \mathbb{R})$ we also have the decomposition

$$
U_{\pi}(g)=\int_{X}^{\oplus} U_{\lambda}(g) d \mu(\lambda) .
$$

If $h_{\pi}$ is the lowest eigenvalue of $L_{0}^{\pi}$ we have by assumption $U_{\pi}(\tilde{r}(2 \pi))=e^{2 \pi i h_{\pi}}$. Hence $U_{\lambda}$ is, for almost every $\lambda$, a positive energy representation satisfying $L_{0}^{\lambda} \geq h_{\pi}$ and $U_{\lambda}(\tilde{r}(2 \pi))=e^{2 \pi i h_{\pi}}$. It follows that, for almost every $\lambda, \pi_{\lambda}$ is an irreducible covariant representation of $\mathcal{A}_{(\mathrm{Vir}, \mathrm{c})}$ with positive energy which, because of Prop. 2.1] is unitarily equivalent to $\pi_{h_{\pi}+n_{\lambda}}^{c}$ for some $n_{\lambda} \in \mathbb{N}_{0}$. Now let $X_{n}=\left\{\lambda \in X: \pi_{\lambda} \simeq \pi_{h_{\pi}+n}^{c}\right\}$. Then, it follows from [35, Lemma 60], that $\left\{X_{n}: n \in \mathbb{N}_{0}\right\}$ is a family of pairwise disjoint measurable subsets of $X$ such that $\mu\left(X \backslash \bigcup_{n \in \mathbb{N}_{0}} X_{n}\right)=0$. Hence

$$
\pi \simeq \bigoplus_{n \in \mathbb{N}_{0}} \int_{X_{n}}^{\oplus} \pi_{\lambda} d \mu(\lambda)
$$


and since $\int_{X_{n}}^{\oplus} \pi_{\lambda} d \mu(\lambda)$ is unitarily equivalent to a (possibly zero) multiple of $\pi_{h_{\pi}+n}^{c}$, (a) follows.

Now it follows from (a) and Prop 2.1 that on the dense subspace $C^{\infty}\left(L_{0}^{\pi}\right)$ of smooth vectors for $L_{0}^{\pi}$ there is a projective representation $\eta$ of the Lie algebra of smooth real vector fields on $S^{1}$ by essentially skew-adjoint operators satisfying $e^{\eta(f)}=\pi_{I}\left(e^{i T_{c}(f)}\right)$ if $I \in \mathcal{J}$ and supp $g \subset I$. Moreover $\eta$ satisfies the assumptions in [53, Theorem 5.2.1] (cf. the proof of [53, Theorem 6.1.1] and the discussion in [38, Appendix]). Hence it can be integrated to a unique strongly continuous projective unitary representation of the covering group of $\operatorname{Diff}^{+}\left(S^{1}\right)$ which, since by assumption $e^{2 \pi i L_{0}^{\pi}}=e^{2 \pi i h_{\pi}}$, factors through $\operatorname{Diff}^{+}\left(S^{1}\right)$ giving a representation $V_{\pi}$ satisfying Eqs. (32) and (34). The remaining claim in (b) then follows easily.

\section{Local extensions}

Definition 3.1. We define a local extension of a conformal net $\mathcal{A}$ to be $a$ conformal net $\left(\mathcal{B}, U, \mathcal{H}_{\mathcal{B}}\right)$ together with a conformal subsystem $\mathcal{C} \subset \mathcal{B}$ such that the corresponding conformal net $\widehat{\mathrm{e}}$ on $\mathcal{H}_{\mathrm{e}}$ is isomorphic to $\mathcal{A}$ and such that

$$
U(\operatorname{PSL}(2, \mathbb{R})) \subset \mathcal{C}\left(S^{1}\right) .
$$

In agreement with the notation for conformal subsystems, since $\mathcal{A}$ and $\widehat{\mathrm{C}}$ are isomorphic, we shall often identify $\mathcal{A}$ and $\mathcal{C}$ and accordingly we shall write $\mathcal{A} \subset \mathcal{B}$ instead of $\mathcal{C} \subset \mathcal{B}$.

Condition (35) implies that $\mathcal{C} \subset \mathcal{B}$ is a full subsystem, namely that

$$
\mathcal{C}\left(S^{1}\right)^{\prime} \cap \mathcal{B}(I)=\mathbb{C} 1 \quad I \in \mathcal{J} .
$$

It prevents trivial extensions of the type $\mathcal{A} \subset \mathcal{A} \otimes \mathcal{C}$, cf. [3]. For finite index subsystems condition (35) is automatically satisfied and we don't know any example of a full conformal subsystem violating it. Note that in the literature the term "local extension" is often used in a weaker sense (see e.g. [44).

A class of examples of local extensions is obtained by considering fix points subsystems under compact group actions. More precisely given a conformal net $\mathcal{B}$ and a strongly compact group $G$ of (vacuum preserving) internal symmetries of $\mathcal{B}$ one can define the fixed point subsystem $\mathcal{A} \equiv$ $\mathcal{B}^{G}$. This kind of construction is paradigmatic in the algebraic approach to quantum field theory, see [15, 18]. 
One has $\mathcal{A}\left(S^{1}\right)^{\prime}=G^{\prime \prime}$ (cf. [18, Theorem 3.6]) and since $U$ and $G$ commute (see [22, Lemma 2.22]), condition (35) is satisfied. Hence $\mathcal{B}$ is a local extension of $\mathcal{A}$ in the sense of Definition 3.1.

If $\pi$ is the identical representation of $\mathcal{A}$ on $\mathcal{H}_{\mathcal{B}}$ one has

$$
\pi=\bigoplus_{\xi \in \hat{G}} d(\xi) \pi_{\xi},
$$

where $\hat{G}$ is set of equivalence classes of irreducible unitary representations of $G$, the $\pi_{\xi}$ are mutually inequivalent irreducible covariant representations of $\mathcal{A}$ (with trivial univalence) appearing with multiplicity $d(\xi)$ equal to the dimension of the representations of $\mathrm{G}$ of class $\xi$ and satisfying $d\left(\pi_{\xi}\right)=d(\xi)$, see [18, 28, 47] and [41, I Sect. 7]. Moreover, the vacuum representation of $\mathcal{A}$ is associated to the trivial one dimensional representation of $G$ and the corresponding Hilbert space $\mathcal{H}_{\mathcal{A}}$ coincides with the subspace of $G$-invariant vectors of $\mathcal{H}_{\mathcal{B}}$.

We denote by $\Delta \equiv \Delta_{\mathcal{B}}$ the semigroup of DHR endomorphism of $\mathcal{A}_{0}$ which are unitarily equivalent to a finite direct sum of representations $\pi_{\xi}, \xi \in \hat{G}$. Then the (DHR) braiding on $\Delta$ gives in fact a permutation symmetry, $\Delta$ is a dual of $G$ in the sense of Doplicher-Roberts duality theory [16, 17] and one can recover the local extension $\mathcal{B}$ by Doplicher-Roberts reconstruction theorem [18, see [47. Prop. 3.8] for the necessary adaptations to conformal nets on $S^{1}$.

More generally, let $\mathcal{A}$ be a conformal net on $S^{1}$ and let $\Delta$ be a semigroup of DHR endomorphisms of $\mathcal{A}_{0}$, all covariant with finite dimension. Assume that the DHR braiding on $\Delta$ is in fact a permutation symmetry (para-Bose statistics for the endomorphisms in $\Delta$ ) and that $\Delta$ is specially directed in the sense of [16, Sect. 5]. Then Doplicher-Roberts construction provides a local extension $\mathcal{B}$ of $\mathcal{A}$ and a strongly compact group $G$ of vacuum preserving internal symmetries of $\mathcal{B}$ such that $\mathcal{A}$ coincides with the fixed point net $\mathcal{B}^{G}$. Note that by [17, Theorem 3.4] (see also [18, Lemma 3.7]) if $\Delta$ has direct sums, subobjects and conjugates then it is specially directed.

In the following we shall use the notation $\mathcal{B}=\mathcal{A} \rtimes \Delta$ for the net obtained through the above Doplicher-Roberts cross product construction.

The decomposition in Eq. (37) suggests the following generalization of the local extensions with compact group action discussed above, cf. [44, Sect. $5]$. 
Definition 3.2. A local extension $\mathcal{B}$ of a conformal net $\mathcal{A}$ is of compact type if the corresponding representation $\pi$ of $\mathcal{A}$ on $\mathcal{H}_{\mathcal{B}}$ satisfies

$$
\pi=\bigoplus n_{i} \pi_{i}
$$

where the $\pi_{i}$ are (necessarily covariant with positive energy) mutually inequivalent irreducible subrepresentations of $\pi$ with finite dimension appearing with multiplicity $n_{i}$ and having finite statistical dimension.

Although we did not assume in Definition 3.2 any bound on the multiplicities $n_{i}$, these turn out to be finite as a consequence of the following proposition, cf. [33, Prop.2.3] and [10] for related results.

Proposition 3.3. Let $\mathcal{B}$ be a local extension of compact type of a conformal net $\mathcal{A}$ on $S^{1}$ and let $\pi$ be the corresponding representation of $\mathcal{A}$ on $\mathcal{H}_{\mathcal{B}}$. Then the following hold:

(a) On $\mathcal{H}_{\mathcal{B}}$ we have

$$
\mathcal{A}(I) \vee \mathcal{A}\left(I^{c}\right)=\mathcal{A}\left(S^{1}\right), \quad I \in \mathcal{J} .
$$

(b) The local extension $\mathcal{B}$ is irreducible, namely

$$
\mathcal{A}(I)^{\prime} \cap \mathcal{B}(I)=\mathbb{C} 1, \quad I \in \mathcal{J} .
$$

(c) Every irreducible representation of $\mathcal{A}$ is contained in $\pi$ with finite (possibly zero) multiplicity.

Proof. Let $\theta$ be a representation of $\mathcal{A}$ localized in $I \in \mathcal{J}$ and equivalent to $\pi$. Then for $J \supset I, \theta_{J}$ is a dual canonical endomorphism for the subfactor $\mathcal{A}(J) \subset \mathcal{B}(J)$. By assumption $\pi$ is a direct sum of covariant representations with finite statistical dimension. Hence we can find isometries $V_{i} \in \mathcal{A}(I), i \in \mathbb{N}$, with orthogonal ranges, satisfying $E_{i}:=V_{i} V_{i}^{*} \in \theta\left(\mathcal{A}\left(S^{1}\right)\right)^{\prime}$, $\sum_{i \in \mathbb{N}} E_{i}=1$ and such that the representations $\sigma^{i}$ defined by $\sigma^{i}(\cdot)=$ $V_{i}^{*} \theta_{J}(\cdot) V_{i}, J \in \mathcal{J}$ are irreducible, covariant, localized in $\mathcal{J}$ and with finite statistical dimension. If $T \in \theta_{I}(\mathcal{A}(I))^{\prime} \cap \mathcal{A}(I)$ then $V_{i}^{*} T V_{j} \sigma^{j}{ }_{I}(\cdot)=\sigma^{i}{ }_{I}(\cdot) V_{i}^{*} T V_{j}$ for $i, j \in \mathbb{N}$ and hence by the equivalence of local and global intertwiners for localized representations with finite dimension [25. Theorem 2.3] we have $V_{i}^{*} T V_{j} \sigma_{J}^{j}(\cdot)=\sigma_{J}^{i}(\cdot) V_{i}^{*} T V_{j}$ for every $J \in \mathcal{J}$. It follows that $E_{i} T E_{j} \in \theta\left(\mathcal{A}\left(S^{1}\right)\right)^{\prime}$ and hence $T \in \theta\left(\mathcal{A}\left(S^{1}\right)\right)^{\prime}$. Since $T \in \theta_{I}(\mathcal{A}(I))^{\prime} \cap \mathcal{A}(I)$ was 
arbitrary and $\theta\left(\mathcal{A}\left(S^{1}\right)\right)^{\prime} \subset \theta_{I}(\mathcal{A}(I))^{\prime} \cap \mathcal{A}(I)$ by Haag duality, we conclude that $\theta\left(\mathcal{A}\left(S^{1}\right)\right)=\theta_{I}(\mathcal{A}(I)) \vee \theta_{I^{c}}\left(A\left(I^{c}\right)\right)$. Hence $\pi\left(\mathcal{A}\left(S^{1}\right)\right)=\pi_{I}(\mathcal{A}(I)) \vee \pi_{I^{c}}\left(A\left(I^{c}\right)\right)$ which proves (a). Now, recalling that $U(\operatorname{PSL}(2, \mathbb{R})) \subset \mathcal{A}\left(S^{1}\right)$, by definition of local extensions, we find $\mathbb{C} 1=\mathcal{A}\left(S^{1}\right)^{\prime} \cap \mathcal{B}(I)$ and hence (b) follows from (a) and locality. Finally (c) follows from [28, p. 39]

Since the defining extensions of the fixed point nets under compact groups of internal symmetries and the finite index extensions are of compact type we can conclude that (b) of Prop. 3.3 generalizes the irreducibility results for conformal subsystems in [7, Prop. 2.1] and [13, Corollary 2.7] (the latter in the local case).

Remark 3.4. If $\mathcal{B}$ is a local extension of compact type of a conformal net $\mathcal{A}$ on $S^{1}$ then it follows from Proposition 3.3 (and its proof) that $\mathcal{A}(I) \subset \mathcal{B}(I)$, $I \in \mathcal{J}$ is an irreducible discrete inclusion of infinite factors in the sense of [28, Definition 3.7].

We now consider the Virasoro net $\mathcal{A}_{(\mathrm{Vir}, 1)}$ with $c=1$. By [49, Prop. 4] $\mathcal{A}_{(\mathrm{Vir}, 1)}$ is the fixed point net under the action of $\mathrm{SO}(3)$ on the conformal net $\mathcal{A}_{\mathrm{SU}(2)_{1}}$ associated to the level one vacuum representation of the loop group $L \mathrm{SU}(2)$. The corresponding representation $\pi$ of $\mathcal{A}_{(\mathrm{Vir}, 1)}$ on $\mathcal{H}_{\mathcal{A}_{\mathrm{SU}(2) 1}}$ satisfies

$$
\pi=\bigoplus_{j \in \mathbb{N}_{0}}(2 j+1) \pi_{j^{2}}^{1}
$$

where $\pi_{j^{2}}^{1}$ is the representation of $\mathcal{A}_{(\mathrm{Vir}, 1)}$ with lowest weight $j^{2}$. As a consequence $d\left(\pi_{j^{2}}^{1}\right)=2 j+1$ for each $j \in \mathbb{N}$ [49, Corollary 6].

We can consider the permutation symmetric semigroup $\Delta$ of DHR endomorphisms of $\mathcal{A}_{(\mathrm{Vir}, 1)}$ which are localized in some $I \in \mathcal{J}_{0}$ and equivalent to a finite direct sum of representations of the type $\pi_{j^{2}}^{1}, j \in \mathbb{N}_{0}$. Then, as discussed above, $\mathcal{A}_{\mathrm{SU}(2)_{1}}$ can be identified with the Doplicher-Roberts cross product $\mathcal{A}_{(\mathrm{Vir}, 1)} \rtimes \Delta$.

Now let $\mathcal{B}$ be a local extension of compact type of $\mathcal{A}_{(\mathrm{Vir}, 1)}$ and let $\pi$ be the corresponding representation of $\mathcal{A}_{(\mathrm{Vir}, 1)}$ on $\mathcal{H}_{\mathcal{B}}$. By Prop. 2.1 and $[8$, Theorem 4.4] every irreducible subrepresentation of $\pi$ is equivalent to a DHR endomorphism in $\Delta$ (note that only subrepresentations with integer lowest conformal energy can appear) and hence

$$
\pi \simeq \bigoplus_{i \in \mathbb{N}} \sigma^{i}
$$


where $\sigma_{i} \in \Delta$, for each $i \in \mathbb{N}$. The local extensions of $\mathcal{A}_{(\mathrm{Vir}, 1)}$ with the above property have been independently classified by the author (cf. the announcement in 32) and by Feng $\mathrm{Xu}$ [55, Sect. 4.2.2]. The resulting possibilities are described in the following theorem (we outline our original proof below).

Theorem 3.5. A local extension $\mathcal{B}$ of $\mathcal{A}_{(\mathrm{Vir}, 1)}$ is of compact type if and only if $\mathcal{B}$ is isomorphic to a fixed point net $\mathcal{A}^{H} \mathrm{SU}(2)_{1}$ for some closed subgroup $H$ of $\mathrm{SO}(3)$.

Proof. The "if part" is a straightforward consequence of the fact that $\mathcal{A}_{\mathrm{SU}(2)_{1}}$ is an extension of compact type of $\mathcal{A}_{(\mathrm{Vir}, 1)}$, cf. Eq. (41). Now let $\mathcal{B}$ be an extension of compact type of $\mathcal{A}_{(\mathrm{Vir}, 1)}$ and let $\pi$ be the corresponding representation of $\mathcal{A}_{(\mathrm{Vir}, 1)}$ on $\mathcal{H}_{\mathcal{B}}$. Given a representation $\theta$ of $\mathcal{A}_{(\mathrm{Vir}, 1)}$ localized in $I \in \mathcal{J}_{0}$ and unitarily equivalent to $\pi$ (so that if $J \supset I, \theta_{J}$ is a dual canonical endomorphism for the inclusion $\left.\mathcal{A}_{(\mathrm{Vir}, 1)}(J) \subset \mathcal{B}(J)\right)$ we deduce from Eq. (42) that $\theta$ is equivalent to (possibly infinite) direct sum of DHR endomorphisms in the permutation symmetric semigroup $\Delta$ defined after Eq. (41). It follows that the monodromy operator $\epsilon_{M}(\rho, \theta):=\epsilon(\rho, \theta) \epsilon(\theta, \rho)$ is trivial (i.e. equal to 1 ) for every $\rho \in \Delta$. We now use the extension of DHR endomorphisms as defined in [44, Prop. 3.9] (cf. also [50, Sect. 3.4.7]) and called $\alpha$-induction in [2]. For every $\rho \in \Delta$, the triviality of the monodromy operator $\epsilon_{M}(\rho, \theta)$ implies that its extension $\alpha_{\rho}$ (we use the notation in [2]) to $\mathcal{B}_{0}$ is still localized in an interval in $\mathcal{J}_{0}$, see [44, Prop. 3.9].

Now the crucial point is that the functorial properties $\alpha$-induction (called homomorphism properties in [2]) imply that $\alpha_{\Delta}$ is still a specially directed permutation symmetric semigroup of Möbius covariant (bosonic) DHR endomorphisms of $\mathcal{B}_{0}$. These functorial properties have been established in [11, 12 for inclusions of local nets on the four dimensional Minkowski spacetime and in 2] for finite index nets of subfactor on the real line.

Due to the triviality of the monodromy (which is automatic in four spacetime dimensions) one can use the arguments in [11, 12, (see also [9, Sect. 2] for an overview) to get the desired structure on $\alpha_{\Delta}$. Hence, as recalled at the beginning of this section, we can use the Doplicher-Roberts cross product construction to define a local extension $\mathcal{B} \rtimes \alpha_{\Delta}$ of the conformal net $\mathcal{B}$.

The next point is that the proof of [12, Theorem 3.5] can be adapted to our situation to show that there is a natural inclusion (up to isomorphism)

$$
\mathcal{A}_{\mathrm{SU}(2)_{1}}=\mathcal{A}_{(\mathrm{Vir}, 1)} \rtimes \Delta \subset \mathcal{B} \rtimes \alpha_{\Delta}
$$


(compatible with $\mathcal{A}_{(\mathrm{Vir}, 1)} \subset \mathcal{B}$ ) and in fact it turns out that $\mathcal{B} \rtimes \alpha_{\Delta}$ is a local extension of $\mathcal{A}_{\mathrm{SU}(2)_{1}}$. But the latter conformal net has no proper local extensions (see e.g. [3] and [33]) and hence we conclude that $\mathcal{B} \rtimes \alpha_{\Delta}=$ $\mathcal{A}_{\mathrm{SU}(2)_{1}}$. Accordingly $\mathcal{B}=\mathcal{A}_{\mathrm{SU}(2)_{1}}^{H}$ for some closed subgroup $H$ of $\mathrm{SO}(3)$ as claimed.

The above proof relies on specific properties of the representation category net $\mathcal{A}_{(\mathrm{Vir}, 1)}$, namely on the fact that the subcategory of representations with finite statistical dimension (and trivial univalence) is permutation symmetric, a fact that appears to be rather exceptional for conformal nets on $S^{1}$. However in the case of local nets on the four dimensional space-time similar ideas have been used by the author and R. Conti to study local extensions in a fairly general context [10]. As matter of fact the above mentioned investigation in [10] inspired our proof of Theorem 3.5.

Coming back conformal nets on $S^{1}$ we remark that there are well known local extensions of the Virasoro net with $c=1$ which are not conformal subsystems of $\mathcal{A}_{\mathrm{SU}(2)_{1}}$ (see e.g. [3] ) and hence are not of compact type as a consequence of Theorem 3.5. However F. Xu has made further progress and classified the local extensions $\mathcal{B}$ of the $c=1$ Virasoro net such that the corresponding representation of $\mathcal{A}_{(\mathrm{Vir}, 1)}$ on $\mathcal{H}_{\mathcal{B}}$ contains a subrepresentation equivalent to some $\pi_{j^{2}}^{1}, j \in \mathbb{N}[55$, Theorem 4.6]. The above condition is called "spectrum condition" in [55] where it is conjectured that all nontrivial extensions of the $c=1$ Virasoro net have to satisfy it. This motivates the following definition

Definition 3.6. A local extension $\mathcal{B}$ of a conformal net $\mathcal{A}$ is maximally non-compact if the corresponding representation $\pi$ of $\mathcal{A}$ on $\mathcal{H}_{\mathcal{B}}$ satisfies the following condition: the only subrepresentation of finite statistical dimension of $\pi$ is the vacuum subrepresentation.

From the previous discussion we can conclude that a local extension of the $c=1$ Virasoro net satisfies Xu's spectrum condition if and only if it is not maximally non-compact. No examples of maximally non-compact extensions of this net seem to be known. We shall however exhibit in Section 5 various examples of maximally non-compact extensions for the Virasoro nets with $c>1$.

We conclude this section with the following proposition.

Proposition 3.7. Let $\mathcal{B}$ be a local extension of the Virasoro net $\mathcal{A}_{(\mathrm{Vir}, c)}$. Then the following hold: 
(a) $\mathcal{A}_{(\mathrm{Vir}, c)}(I)^{\prime} \cap \mathcal{B}(I)=\mathbb{C} 1$, for every $I \in \mathcal{J}$;

(b) The net $\mathcal{B}$ is diffeomorphism covariant.

Proof. Let $\pi$ be the representation of $\mathcal{A}_{(\mathrm{Vir}, c)}$ on $\mathcal{H}_{\mathcal{B}}$ associated with the local extension $\mathcal{B}$. If $V$ is the corresponding strongly continuous projective unitary representation of $\operatorname{Diff}^{+}\left(S^{1}\right)$ on $\mathcal{H}_{\mathcal{B}}$ given by (b) of Prop 2.2 then $V(\gamma) \in \mathcal{A}_{(\mathrm{Vir}, c)}(I)$ if $\gamma \in \operatorname{Diff}(I)$, for each $I \in \mathcal{J}$. Moreover, for every $g \in$ $\operatorname{PSL}(2, \mathbb{R})$ we have $V(g)=p(U(g))$, where $U$ is the representation makes $\mathcal{B}$ Möbius covariant. Hence, it follows from [38, Theorem 12] that

$$
\mathcal{A}_{(\mathrm{Vir}, c)}(I)^{\prime} \cap \mathcal{B}(I)=U(\operatorname{PSL}(2, \mathbb{R}))^{\prime} \cap \mathcal{B}(I)=\mathbb{C} 1
$$

which proves (a).

Now let $I$ be a given interval in $\mathcal{J}$ and let $\gamma \in \operatorname{Diff}^{+}\left(S^{1}\right)$ be such that $\gamma I=I$. Since $\gamma$ preserves the orientation it must keep fixed the boundary points of I. An elementary argument (which we omit here) then shows that for every $J \in \mathcal{J}$ containing the closure of $I$ we can find a diffeomorphism $\gamma^{J} \in \operatorname{Diff}(J)$ with $\left.\gamma^{J}\right|_{I}=\left.\gamma\right|_{I}$, i.e. $\gamma^{-1} \gamma^{J} \in \operatorname{Diff}\left(I^{c}\right)$. Since $V\left(\gamma^{-1} \gamma^{J}\right) \in$ $\mathcal{A}_{(\mathrm{Vir}, c)}\left(I^{c}\right) \subset \mathcal{B}\left(I^{c}\right)$, we find

$$
V\left(\gamma^{J}\right) \mathcal{B}(I) V\left(\gamma^{J}\right)^{*}=V(\gamma) V\left(\gamma^{-1} \gamma^{J}\right) \mathcal{B}(I) V\left(\gamma^{-1} \gamma^{J}\right)^{*} V(\gamma)^{*}=V(\gamma) \mathcal{B}(I) V(\gamma)^{*}
$$

and hence $V(\gamma) \mathcal{B}(I) V(\gamma)^{*} \subset \mathcal{B}(J)$, for every $J \in \mathcal{J}$ containing the closure of $I$. Thus, being conformal nets continuous from outside, we conclude that $V(\gamma) \mathcal{B}(I) V(\gamma)^{*} \subset \mathcal{B}(I)$. If $\gamma$ is arbitrary we can always find a $g \in \operatorname{PSL}(2, \mathbb{R})$ such that $g I=\gamma I$. It follows that

$$
\begin{aligned}
V(\gamma) \mathcal{B}(I) V(\gamma)^{*} & =U(g) V\left(g^{-1} \gamma\right) \mathcal{B}(I) V\left(g^{-1} \gamma\right)^{*} U(g)^{*} \\
& \subset \mathcal{B}(g I)=\mathcal{B}(\gamma I),
\end{aligned}
$$

and hence, for every $I \in \mathcal{J}, \gamma \in \operatorname{Diff}^{+}\left(S^{1}\right)$, we have

$$
V(\gamma) \mathcal{B}(I) V(\gamma)^{*}=\mathcal{B}(\gamma I)
$$

and also (b) is proved.

Remark 3.8. If $\mathcal{B}$ is a diffeomorphism covariant net on $S^{1}$ and $V$ is the corresponding projective unitary representation of $\operatorname{Diff}^{+}\left(S^{1}\right)$ one can define a covariant subsystem $\mathcal{C}$ of $\mathcal{B}$ by

$$
\mathcal{C}(I)=\{V(\gamma): \gamma \in \operatorname{Diff}(I)\}^{\prime \prime} \quad I \in \mathcal{J} .
$$


Arguing as in the proof of Prop. 2.1 it can be shown that the conformal net e on $S^{1}$ is isomorphic to $\mathcal{A}_{(\mathrm{Vir}, c)}$ for some $c \in D \cup[1, \infty)$. It follows that the correspondence between diffeomorphism covariant nets on $S^{1}$ and local extensions of the Virasoro nets is one-to-one, cf. [33].

\section{On the oscillator representations of the $\mathrm{Vi}$ - rasoro nets with $c>1$}

Let $\left(\mathcal{A}_{\mathrm{U}(1)}, U, \mathcal{H}_{\mathrm{U}(1)}\right)$ be the conformal net generated by the $\mathrm{U}(1)$ chiral current algebra, see [3, 5]. The Hilbert space $\mathcal{H}_{\mathrm{U}(1)}$ and the net $\mathcal{A}_{\mathrm{U}(1)}$ can be identified with the Fock space $e^{\mathcal{H}_{1}}$, where $\mathcal{H}_{1}$ is acted on by the irreducible representation of $\operatorname{PSL}(2, \mathbb{R})$ of lowest weight 1 , and with the corresponding second quantization net respectively [26].

We denote $\mathcal{H}_{\mathrm{U}(1)}^{f i n}$ the dense subspace of finite energy vectors, i.e. the algebraic direct sum of the $L_{0}$ eigenspaces. Then $\mathcal{H}_{\mathrm{U}(1)}^{f i n}$ carries the unique irreducible lowest weight representation of the oscillator (Heisenberg) algebra

$$
\begin{array}{r}
{\left[J_{n}, J_{m}\right]=n \delta_{n+m, 0} \quad m, n \in \mathbb{Z},} \\
J_{0}=q 1,
\end{array}
$$

with lowest weight $q=0$, see [3] and [30, Sect. 2.2]. The corresponding lowest weight vector is the vacuum vector $\Omega$ and for $\xi, \psi \in \mathcal{H}_{\mathrm{U}(1)}^{f i n}, n \in \mathbb{Z}$ we have

$$
\left(\xi, J_{n} \psi\right)=\left(J_{-n} \xi, \psi\right),
$$

(hermiticity). Note that defining $J_{n}^{q}:=J_{n}, J_{0}^{q}=q 1$ we obtain a unitary representation of the oscillator algebra with arbitrary lowest weight $q \in \mathbb{R}$.

The $\mathrm{U}(1)$ current $J(z), z=e^{i \vartheta} \in S^{1}$ is defined as an operator valued distribution by

$$
J(z)=\sum_{n \in \mathbb{Z}} J_{n} z^{-n-1}
$$

and the common invariant domain for the smeared field operators

$$
J(u)=\oint_{S^{1}} \frac{d z}{2 \pi i} J(z) u(z) \quad u \in C^{\infty}\left(S^{1}\right)
$$

can be chosen to be the subspace $C^{\infty}\left(L_{0}\right)$ of smooth vectors for $L_{0}$. For a real function $u \in C^{\infty}\left(S^{1}\right), J(u)$ is essentially self-adjoint and the unitary operators $W(u):=e^{i J(u)}$ with $u \in C^{\infty}\left(S^{1}\right)$ real, supp $u \subset I$ generate $\mathcal{A}_{\mathrm{U}(1)}(I)$ 
for every $I \in \mathcal{J}$. Moreover the Weyl relations hold:

$$
W(u) W(v)=W(u+v) \mathrm{e}^{-\oint_{S^{1}} \frac{d z}{4 \pi \imath} u^{\prime}(z) v(z)},
$$

for real smooth functions $u, v$, where $u^{\prime}(z)$ denotes the derivative $\frac{d}{d z} u(z)=$ $-i e^{-i \vartheta} \frac{d}{d \vartheta} u\left(e^{i \vartheta}\right)$.

As shown in [3] (see also [4]) for every $q \in \mathbb{R}$ there is a covariant irreducible representation of $\mathcal{A}_{\mathrm{U}(1)}$ (BMT-automorphism) $\gamma_{q}$ on $\mathcal{H}_{\mathrm{U}(1)}$ such that

$$
\gamma_{q_{I}}(W(u))=e^{i q \oint_{S^{1}} \frac{d z}{2 \pi i} z^{-1} u(z)} W(u)=e^{i J^{q}(u)},
$$

for $I \in \mathcal{J}, u \in C^{\infty}\left(S^{1}\right)$ with support in $I$. Here the field $J^{q}(z)$ is defined by

$$
J^{q}(z)=\sum_{n \in \mathbb{Z}} J_{n}^{q} z^{-n-1}=J(z)+q z^{-1} .
$$

$\gamma_{q_{1}}$ and $\gamma_{q_{2}}$ are inequivalent if $q_{1} \neq q_{2}$. Moreover, if $\varphi$ is a real smooth function such that $-i \varphi^{\prime}(z)=z^{-1} q$ for $z \in I$ then

$$
\gamma_{q_{I}}(\cdot)=\operatorname{Ad} W(-\varphi)(\cdot)
$$

and hence $\gamma_{q}$ is locally implementable by Weyl unitaries. In fact Eq. (51) can be used to define the representation $\gamma_{q}$. Note that $\gamma_{0}$ is the vacuum representation of $\mathcal{A}_{\mathrm{U}(1)}$ and that for every $I \in \mathcal{J}$, we have

$$
\gamma_{q_{I}}\left(\mathcal{A}_{\mathrm{U}(1)}(I)\right)=\mathcal{A}_{\mathrm{U}(1)}(I) .
$$

We now come to the oscillator representations of the Virasoro algebra. For $\lambda, q \in \mathbb{R}, n \in \mathbb{Z}$ the operators

$$
L_{n}^{(\lambda, q)}=\delta_{n, 0} \frac{\lambda^{2}}{2}+\frac{1}{2} \sum_{j \in \mathbb{Z}}: J_{-j}^{q} J_{j+n}^{q}:+i \lambda n J_{n}^{q},
$$

where the colons denote normal ordering, define a positive energy unitary representation $R(\lambda, q)$ of the Virasoro algebra on $\mathcal{H}_{\mathrm{U}(1)}^{f i n}$ with central charge $c=1+12 \lambda^{2}$, see e.g. [30. Sect. 3.4]. Since $L_{0}$ coincides with $L_{0}^{(0,0)}$ (by the Sugawara formula) we have $L_{0}^{(\lambda, q)}=L_{0}+\left(\lambda^{2}+q^{2}\right) / 2$ and hence $\Omega$ is a lowest energy vector for these representations with energy $\left(\lambda^{2}+q^{2}\right) / 2$.

We associate to the above representations the energy-momentum tensors $T^{(\lambda, q)}(z)$ defined by

$$
T^{(\lambda, q)}(z)=\sum_{n \in \mathbb{Z}} L_{n}^{(\lambda, q)} z^{-n-2} .
$$


Then the following holds (see [21, Remark 4.2])

$$
T^{(\lambda, q)}(z)=\frac{1}{2}: J^{q}(z)^{2}:-i \lambda\left(\frac{1}{z}+\frac{\mathrm{d}}{\mathrm{d} z}\right) J^{q}(z)+\frac{\lambda^{2}}{2 z^{2}},
$$

and hence, recalling that $J^{q}(z)=J(z)+q z^{-1}$,

$$
T^{(\lambda, q)}(z)=\frac{1}{2}: J(z)^{2}:+\frac{q}{z} J(z)-i \lambda\left(\frac{1}{z}+\frac{\mathrm{d}}{\mathrm{d} z}\right) J(z)+\frac{\lambda^{2}+q^{2}}{2 z^{2}} .
$$

For $f \in C^{\infty}\left(S^{1}\right)$ the smeared field operator

$$
T^{(\lambda, q)}(f)=\oint_{S^{1}} \frac{d z}{2 \pi i} T^{(\lambda, q)}(z) f(z)
$$

is well defined on the domain $C^{\infty}\left(L_{0}\right)$ and leave it globally invariant. Moreover we see from Eq. (56) that the field $T^{(\lambda, q)}(z)$ is local with respect to $J(z)$ in the sense that if $f, u \in C^{\infty}\left(S^{1}\right)$ have disjoint supports the operators $T^{(\lambda, q)}(f)$ and $J(u)$ commute on $C^{\infty}\left(L_{0}\right)$ and that $T^{(\lambda, q)}(f)$ is hermitian if $f$ is a real function. Finally, it follows from [5, Sect. 2] (cf. also [24]) that $T^{(\lambda, q)}(f)$ is essentially self-adjoint for each real valued smooth function $f$ and that in this case $e^{i T^{(\lambda, q)}(f)}$ commutes with $W(u)$ if the support of the real function $u$ is disjoint from the one of $f$.

We now define an isotonous net $\mathcal{B}^{(\lambda, q)}$ on $\mathcal{H}_{\mathrm{U}(1)}$ by

$$
\mathcal{B}^{(\lambda, q)}(I)=\left\{e^{i T^{(\lambda, q)}(f)}: f \in C^{\infty}\left(S^{1}\right) \text {, real, supp } f \subset I\right\}^{\prime \prime},
$$

for $I \in \mathcal{J}$. As a consequence of the above discussion and of Haag duality for $\mathcal{A}_{\mathrm{U}(1)}$ we obtain the following proposition.

Proposition 4.1. For every $I \in \mathcal{J}$ we have

$$
\mathcal{B}^{(\lambda, q)}(I) \subset \mathcal{A}_{\mathrm{U}(1)}(I) .
$$

The net $\mathcal{B}^{(\lambda, q)}$ so defined it is not in general a conformal subsystem of $\mathcal{A}_{\mathrm{U}(1)}$. And in fact it can be shown that $\mathcal{B}^{(\lambda, q)}$ transforms covariantly with respect to the representation $U$ making $\mathcal{A}_{\mathrm{U}(1)}$ Möbius covariant only for $(\lambda, q)=(0,0), \mathcal{B}^{(0,0)}$ being the $(c=1)$ Virasoro subnet of $\mathcal{A}_{\mathrm{U}(1)}$. However, the equality $L_{0}^{(\lambda, q)}=L_{0}+\left(\lambda^{2}+q^{2}\right) / 2$ implies rotation covariance for every $(\lambda, q) \in \mathbb{R}^{2}$, namely

$$
U(r(\vartheta)) \mathcal{B}^{(\lambda, q)}(I) U(r(-\vartheta))=\mathcal{B}^{(\lambda, q)}(r(\vartheta) I) \quad \vartheta \in \mathbb{R}, I \in \mathcal{J} .
$$

We shall need the following two lemmata. 
Lemma 4.2. For every pair $(\lambda, q) \in \mathbb{R}^{2}$ and every $I \in \mathcal{J}$ the following holds:

$$
\mathcal{B}^{(\lambda, q)}(I)=\gamma_{q_{I}}\left(\mathcal{B}^{(\lambda, 0)}(I)\right)
$$

Proof. Let $\varphi, f \in C^{\infty}\left(S^{1}\right)$ be real functions such that $-i \varphi^{\prime}(z)=z^{-1} q$ for $z \in I$ and $\operatorname{supp} f \subset I$. For $\xi, \psi \in \mathcal{H}_{\mathrm{U}(1)}^{\text {fin }}$ a straightforward calculation shows that

$$
\left(\xi, W(-\varphi) T^{(\lambda, 0)}(f) \psi\right)=\left(T^{(\lambda, q)}(f) \xi, W(-\varphi) \psi\right) .
$$

Since $\mathcal{H}^{\text {fin }}$ is a common core for $T^{(\lambda, 0)}(f)$ and $T^{(\lambda, q)}(f)$ it follows that

$$
W(-\varphi) e^{i T^{(\lambda, 0)}(f)} W(\varphi)=e^{i T^{(\lambda, q)}(f)}
$$

and hence, recalling Eq. (51)

$$
\gamma_{q_{I}}\left(e^{i T^{(\lambda, 0)}(f)}\right)=e^{i T^{(\lambda, q)}(f)},
$$

cf. [5, p. 123] and [4, p. 361]. The conclusion then follows from the definition of $\mathcal{B}^{(\lambda, q)}(I)$ given in Eq. (58).

Lemma 4.3. The representation $R(\lambda, q)$ is irreducible for every $\lambda \neq 0$ and $q \in \mathbb{R}$.

Proof. The character $\chi_{(\lambda, q)}(t), t \in(0,1)$ of the representation $R(\lambda, q)$ is given by

$$
\chi_{(\lambda, q)}(t)=\operatorname{Tr}\left(t^{L_{0}^{(\lambda, q)}}\right)=t^{\frac{\lambda^{2}+q^{2}}{2}} p(t),
$$

where $p(t)=\prod_{n=1}^{\infty}\left(1-t^{n}\right)^{-1}=\operatorname{Tr}\left(t^{L_{0}}\right)$ and hence the conclusion follows since, by [30, Eq. (3.15) and Prop. 8.2], it coincides with the character of the irreducible representation $L(c, h)$ of Vir with central charge $c=1+12 \lambda^{2}$ and lowest weight $h=\left(\lambda^{2}+q^{2}\right) / 2$.

Corollary 4.4. Let $\mathcal{A}_{(\mathrm{Vir}, \mathrm{c})}$ be the Virasoro net with central charge $c=1+$ $12 \lambda^{2}, \lambda \neq 0$ and let $\pi_{h}^{c}$ be the (irreducible) representation of $\mathcal{A}_{(\mathrm{Vir}, c)}$ with lowest weight $h=\left(\lambda^{2}+q^{2}\right) / 2$ as defined in Subsect. 2.4. Then there is a representation $\pi_{(\lambda, q)}$ of $\mathcal{A}_{(\mathrm{Vir}, c)}$ on $\mathcal{H}_{\mathrm{U}(1)}$, unitarily equivalent to $\pi_{h}^{c}$, such that for every $I \in \mathcal{J}$ the following holds:

$$
\pi_{(\lambda, q)}\left(\mathcal{A}_{(\operatorname{Vir}, c)}(I)\right)=\mathcal{B}_{(\lambda, q)}(I) .
$$

We shall need the following proposition in the next section. 
Proposition 4.5. Let $\mathcal{A}_{(\mathrm{Vir}, c)}$ be a Virasoro net with $c>1$. Then, if $h \geq$ $(c-1) / 24$ we have $d\left(\pi_{h}^{c}\right)=d(c)$, where $d(c) \in[1, \infty]$ does not depend on $h$ and satisfies $d(c)>1$.

Proof. The assumption on the range of $c$ and $h$ implies that we can find $\lambda \neq 0$ and $q \in \mathbb{R}$ such that $c=1+12 \lambda^{2}$ and $h=\left(\lambda^{2}+q^{2}\right) / 2$. Then it follows from Corollary 4.4 that $d\left(\pi_{h}^{c}\right)=d\left(\pi_{(\lambda, q)}\right)$ and we have to show that the latter does not depend on $q$.

By Eq. (16) and Corollary 4.4 we find

$$
d\left(\pi_{(\lambda, q)}\right)^{2}=\left[\mathcal{B}_{(\lambda, q)}\left(I^{c}\right)^{\prime}: \mathcal{B}_{(\lambda, q)}(I)\right], \quad I \in \mathcal{J} .
$$

From Proposition 4.1 and Haag duality for $\mathcal{A}_{\mathrm{U}(1)}$ it follows that

$$
\mathcal{B}_{(\lambda, q)}(I) \subset \mathcal{A}_{\mathrm{U}(1)}(I)=\mathcal{A}_{\mathrm{U}(1)}\left(I^{c}\right)^{\prime} \subset \mathcal{B}_{(\lambda, q)}\left(I^{c}\right)^{\prime}
$$

and hence, using the multiplicativity of the minimal index 42] (cf. the proof of [8, Prop. 3.1]), that

$$
d\left(\pi_{(\lambda, q)}\right)^{2}=\left[\mathcal{A}_{\mathrm{U}(1)}(I): \mathcal{B}_{(\lambda, q)}(I)\right] \cdot\left[\mathcal{A}_{\mathrm{U}(1)}\left(I^{c}\right): \mathcal{B}_{(\lambda, q)}\left({ }^{c}\right)\right] .
$$

Now, using Lemma 4.2 and Eq. (52) we find, for an arbitrary $J \in I$,

$$
\begin{aligned}
{\left[\mathcal{A}_{\mathrm{U}(1)}(J): \mathcal{B}_{(\lambda, q)}(J)\right] } & =\left[\gamma_{q_{J}}\left(\mathcal{A}_{\mathrm{U}(1)}(J)\right): \gamma_{q_{J}}\left(\mathcal{B}_{(\lambda, 0)}(J)\right)\right] \\
& =\left[\mathcal{A}_{\mathrm{U}(1)}(J): \mathcal{B}_{(\lambda, 0)}(J)\right]
\end{aligned}
$$

and hence

$$
d\left(\pi_{(\lambda, q)}\right)^{2}=\left[\mathcal{A}_{\mathrm{U}(1)}(I): \mathcal{B}_{(\lambda, 0)}(I)\right] \cdot\left[\mathcal{A}_{\mathrm{U}(1)}\left(I^{c}\right): \mathcal{B}_{(\lambda, 0)}\left(I^{c}\right)\right]
$$

does not depend on $q$.

Finally if $d(c)=1$ then, for every $I \in \mathcal{J}, \pi_{(\lambda, q)}\left(\mathcal{A}_{(\operatorname{Vir}, c)}(I)\right)=\mathcal{A}_{\mathrm{U}(1)}(I)$ which is impossible since $\mathcal{A}_{\mathrm{U}(1)}$ is strongly additive (see [5, 26]) while $\mathcal{A}_{(\mathrm{Vir}, c)}$ it is not.

\section{Sectors with infinite dimension and maxi- mally non-compact local extensions.}

Let $D \subset\left[\frac{1}{2}, 1\right)$ be the set of allowed values of the central charge in the discrete series representations of Vir as defined in Subsect. 2.4 and let $c \in$ 
$(D+1) \cup[2, \infty)$. Then $c-1$ is an allowed value of the central charge and the tensor product net $\mathcal{A}_{(\mathrm{Vir}, c-1)} \otimes \mathcal{A}_{\mathrm{SU}(2)_{1}}$ is a local extension of $\mathcal{A}_{(\mathrm{Vir}, c)}$. The representation of $\mathcal{A}_{(\mathrm{Vir}, 1)}$ on $\mathcal{H}_{\mathcal{A}_{\mathrm{SU}()_{1}}}$ contains the irreducible lowest weight representation $\pi_{j^{2}}^{1}, j \in \mathbb{N}_{0}$ with multiplicity $2 j+1$ (see Eq. (411)) and hence the multiplicity $m(c, j)$ of $\pi_{j^{2}}^{c}$ in the representation of $\mathcal{A}_{(\mathrm{Vir}, c)}$ on $\mathcal{H}_{\mathcal{A}_{(\mathrm{Vir}, c-1)}} \otimes$ $\mathcal{H}_{\mathcal{A}_{\mathrm{SU}(2)_{1}}}$ satisfies $m(c, j) \geq 2 j+1$ for every $j \in \mathbb{N}_{0}$. We are now ready to prove the following theorem, cf. [8, Theorem 4.4] and the guess in [49, Sect. 2].

Theorem 5.1. If $c \in(D+1) \cup[2, \infty)$ and $h \geq(c-1) / 24$ then $d\left(\pi_{h}^{c}\right)=\infty$.

Proof. Let $\pi$ be the representation of $\mathcal{A}_{(\mathrm{Vir}, c)}$ in $\mathcal{H}_{\mathcal{A}_{(\mathrm{Vir}, c-1)}} \otimes \mathcal{H}_{\mathcal{A}_{\mathrm{SU}(2)}}$ as described above. Then, as explained in Sect. 2, $\pi$ is unitarily equivalent to a representation $\theta$ on $\mathcal{H}_{\mathcal{A}_{(\mathrm{Vir}, c)}}$ localized in an interval $I_{0} \in \mathcal{J}$ and for every $I \in \mathcal{J}$ with $I_{0} \subset I \theta_{I}$ is a dual canonical endomorphism for the inclusion $\mathcal{A}_{(\mathrm{Vir}, c)}(I) \subset \mathcal{A}_{(\mathrm{Vir}, c-1)}(I) \otimes \mathcal{A}_{\mathrm{SU}(2)_{1}}(I)$, which is irreducible because of Prop. 3.7. Now let $\rho_{j^{2}}^{c}$ be a representation of $\mathcal{A}_{(\mathrm{Vir}, c)}$ on $\mathcal{H}_{\mathcal{A}_{(\mathrm{Vir}, c)}}$, unitarily equivalent to $\pi_{j^{2}}^{c}$ and localized in $I_{0}$ and let $I \in \mathcal{J}$ be an interval containing $I_{0}$. As shown just before the statement of this theorem the multiplicity $m(c, j)$ of the representation $\rho_{j^{2}}^{c}$ in $\theta$ satisfies $m(c, j) \geq 2 j+1$. Hence (by Haag duality) the endomorphism $\rho_{j^{2} I}^{c}$ is contained in $\theta_{I}$ with multiplicity $n(c, j) \geq 2 j+1$ for each $j \in \mathbb{N}_{0}$. Now, it follows from Prop. 4.5 that $d\left(\rho_{j^{2}}^{c}\right)=d(c)$, for each $j \geq \sqrt{(c-1) / 24}$, where $d(c)$ does not depend on $j$. Let us assume that $d(c)<\infty$. Then by [25, Corollary 2.10] $\rho_{j^{2} I}^{c}$ is an irreducible endomorphism of $\mathcal{A}_{(\mathrm{Vir}, c)}(I)$ for every $j \geq \sqrt{(c-1) / 24}$ and by [28, p. 39] we conclude that $2 j+1 \leq n(c, j) \leq d(c)^{2}$ for every $j \geq \sqrt{(c-1) / 24}$, in contradiction with the assumption $d(c)<\infty$. Hence $d(c)=\infty$ and the conclusion follows from Prop. 4.5,

Corollary 5.2. If $c \in(D+1) \cup[2, \infty)$ and $\mathcal{B}$ is a local extension of compact type of $\mathcal{A}_{(\mathrm{Vir}, c)}$ then the index $\left[\mathcal{B}: \mathcal{A}_{(\mathrm{Vir}, c)}\right]$ is finite.

Proof. Let $\pi$ the representation of $\mathcal{A}_{(\mathrm{Vir}, c)}$ on $\mathcal{H}_{\mathcal{B}}$ defined by the local extension $\mathcal{B}$. Only representations with integer lowest weigh can appear in the decomposition of $\pi$. But there are only a finite number of positive integers $m$ satisfying $m<(c-1) / 24$ and hence, by Theorem [5.1] only a finite number of irreducible DHR sectors can appear in the decomposition of $\pi$. Now, recalling that the inclusion $\mathcal{A}_{(\mathrm{Vir}, c)}(I) \subset \mathcal{B}(I), \quad I \in \mathcal{J}$, is irreducible, the conclusion follows from (the proof of) [33, Prop. 2.3]. 
Now let $G$ be a simply connected compact Lie group with simple Lie algebra $\operatorname{Lie}(G)$ and let $k$ be a positive integer. We denote by $\mathcal{A}_{G_{k}}$ the conformal net associated to the vacuum representation of the corresponding Loop group (or affine Lie algebra) at level $k$ (see [22, 49, 52, 54]). As it is well known, the Sugawara formula (see e.g. [14, Sect. 15.2] and [30, Sect.10.1]), implies that the net $\mathcal{A}_{G_{k}}$ is a local extension of the Virasoro net $\mathcal{A}_{(\mathrm{Vir}, c)}$ with central charge

$$
c \equiv c\left(G_{k}\right)=\frac{\operatorname{dim}(G) k}{k+h^{\vee}},
$$

where $h^{\vee}$ is the dual Coxeter number of $\operatorname{Lie}(G)$, cf. [22, Sect. III.7] and [49, Sect.1]. The central charge $c\left(G_{k}\right)$ is bounded by

$$
r \leq c\left(G_{k}\right) \leq \operatorname{dim}(G),
$$

where $r$ is the rank of $\operatorname{Lie}(G)$ and the lower bound is saturated only for a simply laced Lie algebras at level $k=1$. Note that $c\left(G_{k}\right)<2$ implies that $r=1$ and thus that $G=\mathrm{SU}(2)$. In the latter case we have $c\left(\mathrm{SU}(2)_{k}\right)=$ $3 k /(k+2)$. If $k \geq 4$ we have $c\left(\mathrm{SU}(2)_{k}\right) \geq 2$. The remaining possibilities are $c\left(\mathrm{SU}(2)_{1}\right)=1, c\left(\mathrm{SU}(2)_{2}\right)=1+1 / 2$ and $c\left(\mathrm{SU}(2)_{3}\right)=1+4 / 5$. We summarize the above discussion in the following lemma.

Lemma 5.3. If $G_{k} \neq \mathrm{SU}(2)_{1}$ then $c\left(G_{k}\right) \in(D+1) \cup[2, \infty)$.

Recall that there is a strongly continuous representation of $G$ in the (unitary) group of internal symmetries of $\mathcal{A}_{G_{k}}$ leaving the vacuum invariant. This representation is not in general faithful and its kernel coincides with the (finite) center $Z(G)$ of $G$. It is known that the fixed point net $\mathcal{A}_{G_{k}}^{G}$ satisfies

$$
\mathcal{A}_{(\mathrm{Vir}, c)} \subset \mathcal{A}_{G_{k}}^{G} \subset \mathcal{A}_{G_{k}}, \quad c=c\left(G_{k}\right),
$$

see [49]. In particular, being $G / Z(G)$ infinite, the index $\left[\mathcal{A}_{G_{k}}: \mathcal{A}_{(\mathrm{Vir}, c)}\right]$ is infinite.

Corollary 5.4. If $G_{k} \neq \mathrm{SU}(2)_{1}$ then the local extension $\mathcal{A}_{G_{k}}$ of $\mathcal{A}_{(\mathrm{Vir}, c)}$, $c=c\left(G_{k}\right)$, is not of compact type.

Proof. Due to Lemma 5.3 we can apply Corollary 5.2 and the conclusion follows from

$$
\left[\mathcal{A}_{G_{k}}: \mathcal{A}_{(\mathrm{Vir}, c)}\right]=\infty
$$


The following consequence of Corollary [5.4 has been pointed out by K.H. Rehren in [4] with a different argument based on the comparison of characters.

It can also be proved using [55, Theorem 2.4] and the fact that $\mathcal{A}_{(\mathrm{Vir}, c)}$ is not strongly additive when $c>1$.

Corollary 5.5. If $G_{k} \neq \mathrm{SU}(2)_{1}$, then the inclusion $\mathcal{A}_{(\mathrm{Vir}, c)} \subset \mathcal{A}_{G_{k}}^{G}$ is proper.

The next result shows that maximally non-compact local extensions naturally appear for the Virasoro nets $c>1$.

Proposition 5.6. If $G_{k} \neq \mathrm{SU}(2)_{1}$ and $c=c\left(G_{k}\right) \leq 25$ then $\mathcal{A}_{G_{k}}$ is a maximally non-compact local extension of $\mathcal{A}_{(\mathrm{Vir}, c)}$.

Proof. The representation $\pi$ of $\mathcal{A}_{(\mathrm{Vir}, c)}$ in $\mathcal{H}_{\mathcal{A}_{G_{k}}}$ can only have irreducible subrepresentations with a nonnegative integer lowest weight. Since by assumption $(c-1) / 24 \leq 1$, it follows from Theorem 5.1 that the only subrepresentation $\pi$ with finite dimension is the vacuum representation. Hence the extension is maximally non-compact.

For $\mathrm{SU}(N) h^{\vee}=N$ and hence $c\left(\mathrm{SU}(N)_{k}\right)=k\left(N^{2}-1\right) /(N+k)$ and we see that Prop. [5.6 gives an infinite series of maximally non-compact extensions of the $c>1$ Virasoro nets. Examples are: $\mathrm{SU}(2)_{k}, k>1$; $\mathrm{SU}(3)_{k}, \mathrm{SU}(4)_{k}, \mathrm{SU}(5)_{k}, \mathrm{k}$ arbitrary; $\mathrm{SU}(N)_{1}, 2 \leq N \leq 26$. Actually the same proof of Prop. 5.6, together with Prop. 22.1, gives the following stronger result.

Theorem 5.7. If $c \in(1+D) \cup[2,25]$ then every local extension of the Virasoro net $\mathcal{A}_{(\mathrm{Vir}, c)}$ is maximally non-compact. In particular $\mathcal{A}_{(\mathrm{Vir}, c)}$ has no local extensions of compact type.

\section{A Appendix}

In this appendix we give a differentiability result for the representations of $\operatorname{Diff}^{+}\left(S^{1}\right)$ which is used in the proof of Prop. 2.1. This result as been essentially obtained by T. Loke [40] (cf. also [54] for analogous results for loop groups) and here we consider the necessary modifications we need in this paper. We shall closely follow the discussion in [40, Chap. I].

An element of the group Mob of Möbius transformations of $S^{1}$ is given by a map $z \mapsto \frac{\alpha z+\beta}{\bar{\beta} z+\bar{\alpha}}$, where $\alpha, \beta$ are complex numbers satisfying $|\alpha|^{2}-$ 
$|\beta|^{2}=1$. Mob is a Lie subgroup of $\operatorname{Diff}^{+}\left(S^{1}\right)$ isomorphic to $\operatorname{PSL}(2, \mathbb{R})$. The corresponding Lie subalgebra of $\operatorname{Vect}\left(S^{1}\right)$ is spanned by the vector fields

$$
x:=-\sin \vartheta \frac{d}{d \vartheta}, y:=-\cos \vartheta \frac{d}{d \vartheta}, h:=\frac{d}{d \vartheta},
$$

whose brackets are given by

$$
[h, x]=-y,[h, y]=x,[x, y]=h .
$$

More generally, for each $n \in \mathbb{N}$, the vector fields

$$
x_{n}:=-\frac{1}{n} \sin n \vartheta \frac{d}{d \vartheta}, y_{n}:=-\frac{1}{n} \cos n \vartheta \frac{d}{d \vartheta}, h_{n}:=\frac{1}{n} \frac{d}{d \vartheta}
$$

span isomorphic Lie subalgebras of $\operatorname{Vect}\left(S^{1}\right)$ each associated to a Lie subgroup $\mathrm{Mob}_{n}$ of $\operatorname{Diff}^{+}\left(S^{1}\right)$. Clearly $\mathrm{Mob}_{1}=$ Mob and it is not hard to see that, for each $n>1, \mathrm{Mob}_{n}$ is isomorphic to an n-fold covering of $\operatorname{PSL}(2, \mathbb{R}) \simeq \operatorname{Mob}$ and that the corresponding covering map transforms the one-parameter group $\exp \left(t h_{n}\right)$ into the one-parameter subgroup $r(t)$ of rotations of $\operatorname{PSL}(2, \mathbb{R})$.

Now let $V$ be a strongly continuous projective unitary representation of $\operatorname{Diff}^{+}\left(S^{1}\right)$ on a separable Hilbert space. For every $n \in \mathbb{N}$, the restriction of $V$ to $\mathrm{Mob}_{n}$ lifts to a strongly continuous unitary representation $U_{n}$ of $\widehat{\operatorname{PSL}(2, \mathbb{R})}$. Note that $\exp \left(2 \pi h_{n}\right)^{n}=1$ and hence $U_{n}(\tilde{r}(2 \pi))^{n}=U_{n}\left(\exp \left(2 \pi h_{n}\right)\right)^{n}=\chi_{n} 1$ for a suitable complex number $\chi_{n}$ of modulus one. In particular $U(\tilde{r}(2 \pi))$ has finite spectrum for each $n \in \mathbb{N}$.

Now let $\frac{1}{n} X_{n}, \frac{1}{n} Y_{n}$ and $\frac{i}{n}\left(L_{0}+c_{n}\right), c_{n} \in \mathbb{R}, c_{1}=0$, be the skew-adjoint generators of the one-parameter groups of unitaries $U_{n}\left(\exp \left(t x_{n}\right)\right), U_{n}\left(\exp \left(t y_{n}\right)\right)$, and $U_{n}\left(\exp \left(t h_{n}\right)\right)$, respectively. On the dense subspace $\mathcal{D}_{n} \subset \mathcal{H}$ of $C^{\infty}$ vectors for the representation $U_{n}$ the above operators define a representation of the Lie algebra (67) and hence we have on $\mathcal{D}_{n}$

$$
\left[i L_{0}, X_{n}\right]=-n Y_{n},\left[i L_{0}, Y_{n}\right]=n X_{n},\left[X_{n}, Y_{n}\right]=i n\left(L_{0}+c_{n}\right), n \in \mathbb{N}
$$

If $V$ is a positive energy representation, since the unitary operator $e^{i 2 \pi L_{0}}$ acts as multiplication by a complex number, the spectrum of $L_{0}$ is pure point and every eigenvalue is of the form $h+n$, where $h \geq 0$ is the lowest eigenvalue of $L_{0}$ and $n$ is a nonnegative integer. 
Now let $\mathcal{H}^{\text {fin }}$ be the linear span of the eigenvectors of $L_{0}$. Loke as shown in [40, Sect. I.1] that if a positive energy representation $V$ is such that the eigenspaces of $L_{0}$ are all finite-dimensional, then

$$
\mathcal{H}^{\text {fin }} \subset \bigcap_{n \in \mathbb{N}} \mathcal{D}_{n}
$$

Moreover he proved that the operators $L_{0}, L_{n}:=i Y_{n}-X_{n}$ and $L_{-n}:=$ $i Y_{n}+X_{n}, n \in \mathbb{N}$ define a unitary representation of Vir on $\mathcal{H}^{\text {fin }}$ and that the corresponding energy-momentum tensor

$$
T(z)=\sum_{n \in \mathbb{Z}} L_{n} z^{-n-2}
$$

extends to an operator valued distribution on the subspace of smooth $L_{0}$ vectors such that $T(f)$ is essentially self-adjoint on $\mathcal{H}^{f i n}$ for each $f \in \operatorname{Vect}\left(S^{1}\right)$ and satisfies

$$
p\left(e^{i T(f)}\right)=V(\exp (f)) .
$$

The finite dimensionality of the $L_{0}$ eigenspace is used in 40, to infer that for each $n \in \mathbb{N}$, the representation $U_{n}$ is a direct sum of positive energy representations of $\mathrm{PSL}(2, \mathbb{R})$ and that $\mathcal{D}_{n} \subset \mathcal{H}^{\text {fin }}$. However these facts hold for every positive energy representation $V$, as a consequence of the proposition below (applied to each representation $U_{n}$ ) and hence the results of Loke described above hold (without any essential modification in the proofs) also if the finite dimensionality of the eigenspaces of $L_{0}$ is not assumed. Moreover the representation of Vir on $\mathcal{H}^{\text {fin }}$ so obtained can be seen to be irreducible (and hence unitarily equivalent to some $L(c, h)$, cf. [30, Remark 3.5]) if and only if the corresponding projective representation $V$ of $\operatorname{Diff}^{+}\left(S^{1}\right)$ is irreducible, cf. Lemma 2.2. in [40, Sect. I.2].

Proposition A.1. Let $U$ be a strongly continuous unitary representation of $\mathrm{PSL}(2, \mathbb{R})$ on a separable Hilbert space $\mathcal{H}$ and let $L_{0}$ be the self-adjoint generator of the restriction of $U$ to the lifting $\tilde{r}(t)$ of the one-parameter rotation subgroup of $\mathrm{PSL}(2, \mathbb{R})$. Assume that the spectrum of $L_{0}$ is bounded from below and that the one of $U(\tilde{r}(2 \pi))$ is finite. Then the following hold:

(a) $U$ is a positive energy representation (i.e. $L_{0}$ has a nonnegative spectrum) and it is completely reducible to a direct sum of irreducible subrepresentations. 
(b) Every eigenvector of $L_{0}$ is a smooth vector for the representation $U$.

Proof. If $U$ is assumed to be irreducible then the positivity of the energy follows from the bound on the spectrum of $L_{0}$ as a consequence of the classification of the irreducible representations of $\widetilde{\operatorname{PS}(2, \mathbb{R})}$ [4] (cf. [40, Sect. I.1.3]) and hence the positive energy condition for $U$ follows in general by direct integral decomposition. Then (a) follows e.g. from [36, Lemma 8]. As a consequence there is an increasing sequence $0=n_{1}<n_{2} \ldots$ of nonnegative integers (which is possibly finite) and a decomposition

$$
\mathcal{H}=\bigoplus_{k} \mathcal{H}_{k}
$$

such that the restriction of $U$ to $\mathcal{H}_{k}$ is a (possibly infinite) multiple of an irreducible representation with lowest weight $h+n_{k}$. Hence if $\psi$ is an eigenvector of $L_{0}$ corresponding to the eigenvalue $\lambda$ we can write

$$
\psi=\sum_{n_{k} \leq \lambda-h}\left(\psi_{k}, \psi\right) \psi_{k}
$$

where $\psi_{k} \in \mathcal{H}_{k}$ is a normalized eigenvector of $L_{0}$.

Since every eigenvector of the generator of rotations in an irreducible representation of $\widetilde{\operatorname{PSL}(2, \mathbb{R})}$ is smooth (see e.g. [48, Sect. I.1]), each $\psi_{k}$ is smooth and hence $\psi$ is smooth vector for the representation $U$ so that also (b) is proved.

We can summarize the discussion in this appendix in the following theorem, cf. [40, Sect. I.2.4].

Theorem A.2. Let $V$ be a strongly continuous positive energy projective unitary irreducible representation of $\operatorname{Diff}^{+}\left(S^{1}\right)$ on a (necessarily separable) Hilbert space $\mathcal{H}$. Then $V$ is unitarily equivalent to the unique projective unitary representation $V_{(c, h)}$ which integrates the Vir-module $L(c, h)$ for some $c>0, h \geq 0$. In particular the corresponding generator of rotations $L_{0}$ has finite-dimensional eigenspaces.

Acknowledgements. The author would like to thank R. Conti, S. Köster and R. Longo for discussions, explanations and comments. Theorem 3.5 has been announced at the Miniworkshop "Conformal Field Theory. An Introduction" held in Rome on March 2003. The author thanks the organizers D. Guido and (again) R. Longo for the invitation. 


\section{References}

[1] Bertozzini P., Conti R., Longo R.: Covariant sectors with infinite dimension and positivity of the energy. Comm. Math. Phys. 193 (1998), 471-492.

[2] Böckenhauer J., Evans D.E.: Modular invariants graphs and $\alpha$-induction for nets of subfactors I. Comm. Math. Phys. 197 (1998), 361-386.

[3] Buchholz D., Mack G., Todorov I.T.: The current algebra on the circle as a germ of local field theories. Nucl. Phys. B (Proc. Suppl.) 5B (1988), 20-56.

[4] Buchholz D., Mack G., Todorov I.T.: Localized automorphisms of the U(1)-current. In 31,

[5] Buchholz D., Schulz-Mirbach H.: Haag duality in conformal quantum field theory. Rev. Math. Phys. 2 (1990), 105-125.

[6] Buchholz D.: Introduction to conformal QFT in two dimensions. Unpublished manuscript (1990).

[7] Carpi S.: Classification of subsystems for the Haag-Kastler nets generated by $c=1$ chiral current algebras. Lett. Math. Phys. 47 (1999), 353-364.

[8] Carpi S.: The Virasoro algebra and sectors with infinite statistical dimension. math.OA/0203027 to appear in Ann. H. Poincaré.

[9] Carpi S., Conti R.: Classification of subsystems, local symmetry generators and intrinsic definition of local observables. In: R. Longo (ed.), Mathematical physics in mathematics and physics. Fields Institute Communications Vol. 30, AMS, Providence, RI, 2001, pp.83-103.

[10] Carpi S., Conti R. : in preparation.

[11] Conti R.: Inclusioni di algebre di von Neumann e teoria algebrica dei campi. Ph.D. Thesis, Università di Roma Tor Vergata (1996).

[12] Conti R., Doplicher S., Roberts J. E.: Superselection theory for subsystems. Comm. Math. Phys., 218 (2001), 263-281.

[13] D'Antoni C., Longo R., Radulescu F.: Conformal nets, maximal temperature and and models from free probability. J. Operator Theory 45 (2001), 195-208.

[14] Di Francesco Ph., Mathieu P., Sénéchal D.: Conformal Field Theory. Springer-Verlag, BerlinHeidelberg-New York, 1996.

[15] Doplicher S., Haag, R., Roberts J.E.: Fields, observables and gauge transformations I, II. Comm. Math. Phys. 13 (1969), 1-23; Comm. Math. Phys. 15 (1969), 173-200.

[16] Doplicher S., Roberts J. E.: Endomorphisms of $C^{*}$-algebras, cross products and duality for compact groups. Ann. Math. 130 (1989), 75-119.

[17] Doplicher S., Roberts J. E.: A new duality theory for compact groups. Invent. Math. 98 (1989), $157-218$.

[18] Doplicher S., Roberts J.E.: Why there is a field algebra with a compact gauge group describing the superselection structure in particle physics. Comm. Math. Phys. 131 (1990), 51-107.

[19] Fredenhagen K., Jörß M.: Conformal Haag-Kastler nets, pointlike localized fields and the existence of operator product expansions. Comm. Math. Phys. 176 (1996), 541-554. 
[20] Fredenhagen K., Rehren K.-H., Schroer B.: Superselection sectors with braid group statistics and exchange algebras II. Geometric aspects and conformal covariance. Rev. Math. Phys. Special Issue (1992), 113-157.

[21] Furlan P., Sotkov G. M., Todorov I.T.: Two-dimensional conformal quantum field theory. Riv. Nuovo Cimento 12, N.6 (1989) 1-202.

[22] Gabbiani F., Fröhlich J.: Operator algebras and conformal field theory. Comm. Math. Phys. 155 (1993), 569-640.

[23] Goddard P., Kent A., Olive D.: Unitary representations of the Virasoro and super-Virasoro algebra. Comm. Math. Phys. 103 (1986), 105-119.

[24] Goodman R. and Wallach N. R.: Projective unitary positive-energy representations of Diff $\left(S^{1}\right) . J$. Funct. Anal. 63, (1985) 299-321.

[25] Guido D., Longo R.: The conformal spin and statistic theorem. Comm. Math. Phys. 181 (1996), $11-35$.

[26] Guido D., Longo R., Wiesbrock H.-W.: Extensions of conformal nets and superselection structures. Comm. Math. Phys. 192 (1998), 217-244.

[27] Haag R.: Local Quantum Physics. 2nd ed. Springer-Verlag, Berlin-Heidelberg-New York, 1996.

[28] Izumi M., Longo R., Popa S.: A Galois correspondence for compact groups of automorphisms of von Neumann algebras with a generalization to Kac algebras. J. Funct. Anal. 155 (1998), 25-63.

[29] Jones V.: Index of subfactors. Invent. Math. 72 (1983), 1-25.

[30] Kac V. G., Raina A. K.: Bombay Lectures on Highest Weight Representations of Infinite Dimensional Lie Algebras. World Scientific, Singapore, 1987.

[31] Kastler D. ed.: The algebraic theory of superselection sectors. World Scientific, Singapore, 1990.

[32] Kawahigashi Y.: Classification of operator algebraic conformal field theories. math.OA/0211141

[33] Kawahigashi Y., Longo R.: Classification local conformal nets. Case $c<1$. math.OA/0211141 to appear in Ann. Math.

[34] Kawahigashi Y., Longo R.: Classification of two-dimensional local conformal nets with $c<1$ and 2-cohomology vanishing for tensor categories. math-ph/0304022

[35] Kawahigashi Y., Longo R., Müger M.: Multi-interval subfactor and modularity of representations in conformal field theory. Comm. Math. Phys. 219 (2001), 631-669.

[36] Köster S.: Conformal transformations as observables. Lett. Math. Phys. 61 (2002), 187-198.

[37] Köster S.: Absence of stress energy tensor in $\mathrm{CFT}_{2}$ models. math-ph/0303053

[38] Köster S.: Local nature of cosets models. math-ph/0303054

[39] Kosaki H.: Extension of Jones' theory on index to arbitrary subfactors. J. Funct. Anal. 66 (1986), 123-140.

[40] Loke T.: Operator algebras and conformal field theory of the discrete series representation of $\operatorname{Diff}^{+}\left(S^{1}\right)$. PhD Thesis, University of Cambridge, 1994. 
[41] Longo R.: Index of subfactors and statistics of quantum fields. I. Comm. Math. Phys. 126 (1989), 217-247, and II. Correspondences, braid group statistics and Jones polynomial. Comm. Math. Phys. 130 (1990), 285-309.

[42] Longo R.: Minimal index and braided subfactors. J. Funct. Anal. 109 (1992), 98-112.

[43] Longo R.: Conformal subnets and intermediate subfactors. Comm. Math. Phys. 237 (2003), 7-30.

[44] Longo R., Rehren K.-H.: Nets of subfactors. Rev. Math. Phys. 7 (1995), 567-597.

[45] Mack G.: Introduction to conformal invariant quantum field theory in two and more dimensions. In G. t' Hooft et al. Eds.: Non perturbative quantum field theory. Plenum Press, New York, 1988, pp.353-383.

[46] Milnor J.: Remarks on infinite-dimensional Lie groups. In B.S. De Witt and R. Stora Eds.: Relativity, groups and topology II. Les Houches, Session XL, 1983, Elsevier, Amsterdam, New York, 1984, pp. 1007-1057.

[47] Müger M.: On charged fields with group symmetry and degeneracies of Verlinde's matrix S. Ann. Inst. H. Poincaré 71 (1999), 359-394.

[48] Pukánzsky L.: The Pancherel formula for the universal covering group of $\operatorname{SL}(2, \mathbb{R})$. Math. Annalen 156 (1964), 96-143.

[49] Rehren K.-H.: A new view of the Virasoro algebra. Lett. Math. Phys. 30 (1994), 125-130.

[50] Roberts J.E.: Lectures on algebraic quantum field theory. In [31, pp. 1-112.

[51] Takesaki M.: Theory of operator algebras I. Springer-Verlag, Berlin-Heidelberg-New York, 2002.

[52] Toledano Laredo V.: Fusion of positive energy representations of $L \operatorname{Spin}_{2 n}$, PhD Thesis, University of Cambridge, 1997.

[53] Toledano Laredo V.: Integrating unitary representations of infinite-dimensional Lie groups, $J$. Funct. Anal. 161 (1999), 478-508.

[54] Wassermann A.: Operator algebras and conformal field theory III: Fusion of positive energy representations of SU(N) using bounded operators. Invent. Math. 133 (1998) 467-538.

[55] Xu F.: Strong additivity and conformal nets. math.QA/0303266 